\title{
Assessing the concept of structure sensitivity or insensitivity for sub-nanometer catalyst materials
}

\author{
Andrew S. Crampton', Marian D. Rötzer ${ }^{1}$, Claron J. Ridge ${ }^{1,2}$, Bokwon Yoon ${ }^{3}$, \\ Florian F. Schweinberger ${ }^{1}$, Uzi Landman ${ }^{3}$ and Ueli Heiz ${ }^{1^{*}}$
}

${ }^{1}$ Lehrstuhl für Physikalische Chemie, Catalysis Research Center \& Chemistry Department, Technische Universität München, Lichtenbergstraße 4, 85748 Garching, Germany

${ }^{2}$ Air Force Research Laboratory, Energetic Materials Branch 2306 Perimeter Rd.

Eglin AFB, 32542, USA

${ }^{3}$ School of Physics, Georgia Institute of Technology, Atlanta, GA 30332-0430, USA

\begin{abstract}
The nature of the nano-catalyzed hydrogenation of ethylene, yielding benchmark information pertaining to the concept of structure sensitivity/insensitivity and its applicability at the bottom of the catalyst particle size-range, is explored with experiments on size-selected Ptn $(n=7-40)$ clusters softlanded on $\mathrm{MgO}$, in conjunction with first-principles simulations. As in the case of larger particles both the direct ethylene hydrogenation channel and the parallel hydrogenation-dehydrogenation ethylidyne- producing route must be considered, with the fundamental uncovering that at the $<1 \mathrm{~nm}$ size-scale the reaction exhibits characteristics consistent with structure sensitivity, in contrast to the structure insensitivity found for larger particles. In this size-regime, the chemical properties can be modulated and tuned by a single atom, reflected by the onset of low temperature hydrogenation at $T$ $>150 \mathrm{~K}$ catalyzed by $\mathrm{Pt}_{\mathrm{n}}(\mathrm{n} \geq 10)$ clusters, with maximum room temperature reactivity observed for $\mathrm{Pt}_{13}$ using a pulsed molecular beam technique. Structure insensitive behavior, inherent for specific cluster sizes at ambient temperatures, can be induced in the more active sizes, e.g. $\mathrm{Pt}_{13}$, by a temperature increase, up to $400 \mathrm{~K}$, which opens dehydrogenation channels leading to ethylidyne formation. This reaction channel was, however found to be attenuated on $\mathrm{Pt}_{20}$, as catalyst activity remained elevated after the $400 \mathrm{~K}$ step. $\mathrm{Pt}_{30}$ displayed behavior which can be understood from extrapolating bulk properties to this size range; in particular the calculated d-band center. In the non-scalable subnanometer size regime, however, precise control of particle size may be used for atom-by-atom tuning and manipulation of catalyzed hydrogenation activity and selectivity.
\end{abstract}

Keywords: Platinum, ethylene hydrogenation, model catalysis, structure sensitivity, clusters 


\section{Introduction}

Heterogeneous catalysis on surface-supported sub-nanometer size-selected clusters has been shown to exhibit unique characteristic trends that portray dependencies on particle size extending down to the limit of differences in chemical activity caused by size-differences entailing one or two atoms [1-4]. Such observations signal the emergence of a size-regime, where trends observed for extended surfaces and particles of larger sizes, and concepts deduced from such observations, cease to apply, or require essential modifications. In this non-scalable size-regime, small is different in an essential way, and scaling of physical and chemical properties of larger particles to those in the small-size domain, as well extrapolations to small sizes of knowledge gained at larger size-scales, are questionable, and often invalid. In light of ever-growing efforts aimed at the development and characterization of nano-scale catalysts, it is imperative that research endeavors be directed at critical assessment, modification, and reformulation of concepts used in catalysis research and their validity, when approaching the bottom of the catalysts' size scale. The time-honored, commonly used, concept of structure sensitivity/insensitivity of a catalyzed reaction is a fundamental concept in heterogeneous catalysis, and in this paper we explore it's applicability at the nanoscale. To this end we investigate here experimentally and through first-principles theory simulations, ethylene hydrogenation on sizeselected, sub-nanometer-size model platinum cluster catalysts deposited on a single-crystal magnesia surface under ultra-high vacuum (UHV) conditions. Prior to exposition of our investigations we review in the following the development of the structure sensitivity/insensitivity concept over the past half century. This is the period that saw the emergence of our beloved late friend and colleague John T. Yates Jr. as a major force in surface science and heterogeneous catalysis research, first at the then National Bureau of Standards, NBS (now the National Institute for Standards and Technology, NIST), later at the University of Pittsburgh, and last at the University of Virginia). This article celebrates John's magnificent contributions to science and society and is dedicated to his memory.

Since the 1960's, heterogeneously catalyzed reactions on transition metals displaying an activity which was dependent on particle size were classified as "demanding" and those which were independent of particle size were termed "facile" [5-7]. This focus on particle size was based on the fact that the number of atoms exposed on a particle surface compared to the total number of atoms within the particle changes drastically from very small sizes of only tens of atoms to bulk like 
structures. Quantifying this relation led to the terms "fraction of exposed atoms" [8] or "degree of dispersion" [5] (varying with particle size, approaching unity for very small particles with diameter s $1 \mathrm{~nm}$ and taking small values for large particles), and this has been a central guiding theme of research in catalysis for over half a century [8-11]. The application of simple geometric (polyhedral) models initiated the line of reasoning that specific configurations of atoms at metal surfaces were responsible for the varying activities. Indeed, steps, corners and edges, i.e. low coordination sites have all been used extensively to explain varying rates of catalytic reactions [5, 8-10]. This line of reasoning has its origins in the concept of active sites introduced in 1925 by Taylor, who in the course of investigating formation of nickel carbonyl on a heterogeneous nickel surface concluded that "only a small fraction of the surface is active" and that "the amount of surface which is catalytically active is determined by the reaction catalyzed" [12]. The above led to introduction of the "Taylor ratio" (TR) that gives for a particular reaction the fraction of active sites relative to the total number of sites on the surface of the catalyst; clearly, a value of $T R=1$ corresponds to a facile reaction, whereas $T R<1$ indicates a demanding one.

The proliferation of atomic and molecular scale surface science preparation and characterization techniques in the 1970 s and onward, focused attention on reactions catalyzed on well-defined single-crystal surface model systems. The classification of reactions as facile or demanding gave way to the more practical terminology of structure-sensitive and structureinsensitive, respectively, and this nomenclature continues to be used to this day.

Accumulated evidence from electron and vibration spectroscopies, time-resolved reaction dynamics studies and first-principles quantum-mechanical electronic structure calculations, brought about extensions of the above classification to include factors that reach beyond the pure morphological and structural effects discussed above. These extensions include: (i) size-effects that influence the electronic structure of the catalyst particle - that is energy level organization and spectra of the valence electrons, particularly the frontier orbitals participating in reactive bond-breaking and bond-making and their evolution in the course of a catalyzed reaction, and (ii) temporal effects pertaining to the dynamics of structural reorganization processes and/or the occurrence of reaction intermediates. The latter extension identifies structure sensitive reactions as being those where the time-scale of such dynamical effects are commensurate with the dynamical processes involved in the catalyst's restructuring and/or in the evolution of certain reaction intermediates, whereas for structure 
insensitive reaction these dynamic processes are either absent, or may occur on a time-scale that leaves the outcome of the reaction unaffected. The most prominent example of a structure sensitive reaction is the ammonia synthesis through the reaction of nitrogen and hydrogen on iron, while ethylene hydrogenation catalyzed by platinum serves as an example of a structure insensitive reaction [13]. The latter fundamental and technological important reaction stands out as the first reaction for which a catalytic mechanistic pathway has been formulated (by Horiuti and Polanyi in 1934), and it forms the focus of our investigation in this paper [14].

The Horiuti-Polanyi (HP) mechanism begins with hydrogen dissociation on the metal catalyst surface and following alkene (ethylene) adsorption hydrogen additions occur in two subsequent steps: first converting the alkene $\left(\mathrm{C}_{2} \mathrm{H}_{4}\right)$ into a "half-hydrogenated" state (ethyl, $\left.-\mathrm{CH}_{2} \mathrm{CH}_{3}\right)$ followed by a second hydrogenation to form the alkane (ethane, $\mathrm{C}_{2} \mathrm{H}_{6}$ ) product which desorbs from the surface. Sum-frequency generation spectroscopy on single crystal surfaces demonstrated that two strongly bound species are present during the catalytic reaction on $\mathrm{Pt}(111)$ and $\mathrm{Pt}(100)$ surfaces (although with different relative concentrations) [13]: (i) di- $\sigma$ ethylene where the carbon atoms of the adsorbed $\mathrm{C}_{2} \mathrm{H}_{4}$ molecule are $\sigma$-bonded (in near $\mathrm{sp}^{3}$ hybridization) to neighboring Pt surface atoms (where two C-Pt bonds anchor the molecule to the surface), and (ii) ethylidyne $\left(\equiv \mathrm{C}-\mathrm{CH}_{3}\right)$, a molecule which may form through several pathways (for a recent discussion see [15]). In this study we favor an ethylidyne formation reaction scheme that starts from adsorbed di- $\sigma$ ethylene and proceeds through hydrogenation-dehydrogenation reactions with formation of the ethyl molecule (via hydrogenation) followed by two dehydrogenations: the first one converting $\mathrm{CH}_{2} \mathrm{CH}_{3}$ to ethylidene $\left(\mathrm{CHCH}_{3}\right)$ and the second yielding ethylidyne. Di- $\sigma$ ethylene and ethylidyne occupy three-fold hollow surface sites (involving reorganization of the neighboring metal atoms); on $\mathrm{Pt}(111)$ ethylidyne is readily formed at around $280 \mathrm{~K}$, and both it, and di- $\sigma$ adsorbed ethylene, are found on all platinum surfaces as stagnant spectators with respect to the hydrogenation reaction.

The structure-insensitive hydrogenation of the ethylene molecule (found in studies on extended platinum surfaces to occur at higher hydrogen pressure and temperature) involves weaklybound $\pi$-bonded ethylene (in a near $\mathrm{sp}^{2}$ hybridization) and the "half-hydrogenated" ethyl $\left(\mathrm{C}_{2} \mathrm{H}_{5}\right)$ molecule as reaction intermediates $[13,16,17]$; for a proposed reaction scheme see Fig. 13 in [11]. To summarize: current opinion is that while the adsorption of ethylene is structure sensitive, the overall hydrogenation reaction is structure insensitive. 
The identification of ethylidyne as a stagnant spectator was shown first by Beebe and Yates in 1986 [18]. In that study, a combined IR and kinetic analysis of the in situ surface concentration of ethylidyne during ethylene hydrogenation was performed on a $\mathrm{Pd} / \mathrm{Al}_{2} \mathrm{O}_{3}$ catalyst and the results uncovered that the formation and hydrogenation of ethylidyne was 2-3 orders of magnitude slower than that of ethylene. They also observed that the reaction rate was unchanged on a clean or ethylidyne pre-covered catalyst. This served as a critical observation in demonstrating that the reaction mechanism did not involve a proposed ethylidyne-ethylidene conversion as a reaction intermediate - rather, ethylidyne has been found to act purely as an inactive spectator.

It has been noted on several occasions in the literature that the application of the nomenclature of structure insensitivity is not without its detractors, and critique of this concept has appeared. In particular, two major questions have been posed: (i) without testing all particle sizes down to a single atom how can a reaction be unequivocally labeled as structure insensitive (especially when much smaller particles are predicted to behave differently [19]), and (ii) what are the local origins of this structure insensitivity on the metal catalyst. A common explanation for (ii) is that carbonaceous species (in particular the aforementioned ethylidyne) populate the surface and mask structural features that may be expressed on different surfaces and that potentially could underlie different reaction outcomes, i.e. the reaction would exhibit structural sensitivity if the surfaces were left naked. These two issues with classifying a reaction as structure insensitive have been pointed out in review articles spanning the course of almost thirty years $[10,11,19,20]$.

This line of reasoning concerning the methodology of deciding whether a reaction catalyzed by a given metal is demanding (structure sensitive) follows the one outlined in [19], where it is proposed that: "The best way to proceed is to choose a molecule reacting along two parallel paths and measure the selectivity defined as the rate of the two parallel reactions. If the two products come from different adsorbed states requiring different surface structures, a change of selectivity with dispersion or mode of preparation of the metal may be found. The most unequivocal case is when the specific activity for one of the parallel reactions changes from one catalyst to the next, while the specific activity for the other remains unchanged". In the following we refer to the above as the: "parallel reactions selectivity criterion" (PRSC).

Real catalysis entails typically highly dispersed small particles supported on metal oxides or other high-surface-area substrates, whereas the results that were reviewed above were all obtained 
from investigations on extended single crystal metal surfaces. To bridge the so called "material-gap" attention has been shifted over the past decade to investigations involving finite particles on solid supports [21, 22]. It is pertinent to remark here that the intrinsic size-effect of platinum particles supported on amorphous alumina in the hydrogenation of ethylene has been previously addressed [23]. In this investigation it was found that the reaction on Pt particles larger than $1.7 \mathrm{~nm}$ was structure insensitive. A maximum of the turn over frequency (TOF) was found for particle size of $\sim 0.6 \mathrm{~nm}$ (containing 10-20 atoms), with a similar result found for a $\mathrm{Pt} / \mathrm{SiO}_{2}$ system, indicating an insignificant particle-support interaction. Moreover the catalytic activity essentially vanished for smaller particles (less than about 10 atoms). From these results it has been suggested that underlying the apparent structure sensitivity at small sizes, and in particular the increased activity, is "increased atom accessibility"; this interpretation has been reached, in the absence of realistic quantitative estimates, on the basis of analysis that considered rather idealized simple polyhedral models and heuristic arguments. Additionally the above experiment has been carried out on polydispersed particle samples, and a deconvolution of the effect of the particle-size distribution could not be made unambiguously; we note that this aspect is shared also by later work (some extending up to this date) involving supported particles, and it acts as an obstacle in attempting a conclusive interpretation of the data and for gaining a deeper understanding of the physical principles governing the observed behavior.

Several studies on the catalytic properties of Pt particles have been reported in the past few years, [see refs. 11-18 in [24]] finding structure insensitivity for ethylene hydrogenation on particles in the range of $1-11 \mathrm{~nm}$, with a TOF $=11.9$ molecule ethane/Pt/s determined for $\mathrm{C}_{2} \mathrm{H}_{4}$ hydrogenation on $1.5 \mathrm{~nm}$ Pt nanoparticles, in excellent agreement with the value measured on platinum single crystal surfaces.

\section{Reactions Catalyzed by Supported Nanoclusters}

The structure-insensitivity of olefin hydrogenation reactions has been challenged in the past several years based on measurements performed on supported Pd nanoparticles. It was found that the hydrogenation activity is enhanced compared to that of extended single crystals for Pd particles with diameters less than $10 \mathrm{~nm}[25,26]$. However, it has been concluded that the catalytic activity is independent of particle size in the range of 1-3 nm and that particles with well-ordered (111) facets 
seem to be less active $[25,27,28]$. These effects have been attributed to a volume effect, whereby hydrogen diffuses deep into the bulk of a single crystal thereby rendering it inaccessible for alkenes adsorbed on the surface, whereas this depletion does not occur on particles of nanoscale dimensions. It is pertinent to note however that the possible participation of sub-surface hydrogen in the reaction is unique to palladium because of its ability to absorb hydrogen, whereas bulk hydrogen is energetically unstable in most metals. Additional effects observed in measurements performed on non-sizeselected distributions of $\mathrm{Pd}$ particles supported on $\mathrm{Al}_{2} \mathrm{O}_{3} / \mathrm{NiAl}(110)$ have led to a suggested sizedependent redistribution between weakly $\pi$-bound and more strongly bound di- $\sigma$ ethylene, with the latter preferentially adsorbed on the larger particles - however, experimental or theoretical evidence has not been provided for specific sizes of the catalytic particles [27].

Understanding and potential utilization of the size-dependent evolution of the physical and chemical properties of materials aggregates, from the molecular and cluster regime to the condensed matter phase are outstanding challenges of modern materials science, with investigations starting in the late 1990's on size-selected supported nanometer-scale metal cluster catalysts heralding the emergence of nanocatalysis as a promising direction in heterogeneous catalysis [1, 3, 29].

At this time, it was Yates, who was very supportive for the field and published together with one of the authors of this work and coworkers at the EXXON Research and Engineering Company the first paper on truly size-selected platinum clusters deposited onto a support from a cluster beam in UHV [30]. In this work, the cluster materials were investigated using $\mathrm{CO}$ adsorption/desorption measurements and comparing the desorption profiles to those obtained from single crystal surfaces. These investigations led to the identification of an adsorption site found only on clusters. This work began what has become a well-established research field of investigating the catalytic properties of size-selected clusters on thin metal-oxide films [1].

Materials in the nanoscale size-range are characterized by discrete quantized energy level spectra, specific structural motifs, unique dynamical properties, and non-scalable size-evolutionary patterns where "each atom counts", and "small is different" in an essential way. Consequently, for materials of nanometer dimensions the very concept of structure sensitivity, or the lack thereof which, as previously mentioned, had its early origins in size-dependence, or independence, of catalyzed reaction rates - may be rendered rather ambiguous and elusive. This difficulty is exacerbated, particularly at this size regime, by inextricable coupling between the clusters' electronic 
and atomic (geometric, or morphological) structures, as well as by the proliferating abundance of close-in-energy cluster isomeric atomic arrangements, occurring with a thermodynamic (Boltzmann distribution) frequency, or induced by the evolving chemical reaction (so-called "structural fluxionality") [31]. Moreover, with decreasing size, changes in electronic properties brought about by charge transfer to, or from, the underlying support (expressed sometime as change in the partial oxidation state of the cluster) and atomic structural variations of the cluster due to bonding and adhesive interactions with the substrate (including dimensionality crossover from a three-dimensional, 3D, nanostructure to a "wetting" two-dimensional, 2D, adsorbed island), occur inevitably, with a varying degree, depending on the cluster and support composition, commensurability, and other physical characteristics (e.g., thickness of the underlying metal-oxide support) [32-35].

Indeed, when the reduction in the size of metal particles reaches the ultimate nanocluster regime, that is, clusters comprised of about 10 to 20 atoms ( $<1 \mathrm{~nm}$ in "diameter"), they may be viewed as quasi-zero-dimensional (OD) quantum dots, whose physical and chemical properties are dominantly affected by quantum size effects $[29,36]$ originating from electron confinement, and where extrapolations from larger sizes using arguments relying on surface-to-volume ratios and scaling relations based on the enumeration of special sites (e.g., corner and edge atoms) as a function of the size are not operative [37-39]. In this size scale - that is, in the limit of ultimate dispersion - where almost all of the atoms of the metal cluster are essentially surface atoms and where almost all the cluster atoms can be classified as under-coordinated (compared to the bulk), a new materials' size-regime is entered. In this limiting reduced-size scale novel emerging properties are expected, requiring experiments and first-principles theoretical treatments with atom-by-atom resolution. This is indeed the approach that we have taken in this study.

Guided by the above observations we pursue our quest pertaining to the applicability of the concept of structure sensitivity (or the lack thereof) to reactions catalyzed by nanoscale cluster model catalysts, by focusing our investigations on the hydrogenation reaction of ethylene catalyzed by sizeselected platinum nanoclusters containing between 7 to $40 \mathrm{Pt}$ atoms supported on an inert $\mathrm{MgO}(100) / \mathrm{Mo}(100)$ surface under UHV conditions. As previously mentioned, ethylene hydrogenation serves as a generic example of a structure insensitive reaction, obeying the "parallel reactions selectivity criterion" [5] (with the half-hydrogenation to ethyl/dehydrogenation to ethylidyne channel and the competing pathway corresponding to full-hydrogenation-to-ethane, serving as the operative 
two parallel reactions). Thus, study of this reaction catalyzed by nanoscale clusters with atom-byatom size-controlled resolution is expected to provide benchmark results pertaining to the reaction's structure-sensitivity/insensitivity nature at the bottom of the catalyst particle size-range [40]. 


\section{Experimental}

Sample and cluster preparation and characterization : All experiments were performed in a UHV chamber with a base pressure of $1 \times 10^{-10} \mathrm{mbar}$ [41]. The Mo(100) (MaTeck, Germany, $0.785 \mathrm{~cm}^{2}$ ) single crystal was cleaned by heating to $2000 \mathrm{~K}$ and oxidation at $900 \mathrm{~K}$ in a $5 \times 10^{-7} \mathrm{mbar}_{2}$ background (5.5 purity, Air Liquide Germany). The purity of the crystal was checked with Auger electron spectroscopy (AES) and ultraviolet photoelectron spectroscopy (UPS) [42]. The MgO(100) thin film was then grown at $600 \mathrm{~K}$ on the $\mathrm{Mo}(100)$ single crystal by evaporating a magnesium ( $\geq 99.95 \%$, Merck Germany) ribbon in front of the crystal in a $5 \times 10^{-7} \mathrm{mbar}$ background of $\mathrm{O}_{2}$. After annealing the film at $800 \mathrm{~K}$ for $\mathrm{t}>10 \mathrm{~min}$., the film was characterized with AES and is estimated to have a thickness of ten atomic layers, the purity and lack of defect sites was further confirmed with temperature programmed desorption (TPD), AES, UPS and metastable impact electron spectroscopy (MIES).

The $\mathrm{Pt}(111)$ (MaTeck, Germany, $0.785 \mathrm{~cm}^{2}$ ) single crystal was cleaned by cycles of argon ion sputtering at $900 \mathrm{~K}$, followed by oxidation for five minutes at $650 \mathrm{~K}$ in a $5 \times 10^{-7} \mathrm{O}_{2}$ background pressure and annealing at $1300 \mathrm{~K}$ for one minute. The purity was checked with AES, UPS and MIES.

The clusters were generated using a laser evaporation source; a detailed description can be found elsewhere [41]. In brief, the $2^{\text {nd }}$ harmonic of a Nd:YAG laser (InnoLas DPSS, $532 \mathrm{~nm}, 100 \mathrm{~Hz}$ ) is focused onto a rotating platinum target (99.95\% purity, Alfa-Aesar Germany), with each pulse being thermalized and extracted into the vacuum with a delayed pulse of helium gas (6.0 purity, Westfalen Germany). Electrostatic lenses guide the clusters to a bender where the cationic species are guided into a quadrupole mass spectrometer (QMS) (Extrel, USA, $16000 \mathrm{amu}$ ). The cluster beam is then size-selected and a single cluster size is deposited onto the $\mathrm{MgO}(100) / \mathrm{Mo}(100)$ substrate. Retarding field analyses ensure that the clusters have a kinetic energy of less than than $1 \mathrm{eV} /$ atom for softlanding conditions. The clusters are neutralized by electron tunneling through the $\mathrm{MgO}$ film and the resulting current is recorded and integrated in order to calculate a cluster coverage. It is assumed that each cluster has unit charge. All TPR curves shown were performed on 0.1 clusters $/ \mathrm{nm}^{2}$ for $\mathrm{Pt}_{7}$ $\mathrm{Pt}_{13}$, and larger sizes on coverages of $0.05-0.08$ clusters $/ \mathrm{nm}^{2}$. The majority of the pulsed molecular beam experiments were also performed on 0.1 clusters $/ \mathrm{nm}^{2}$ and extrapolating data measured on lower coverages did not produce any significant variations in the turnover frequency. 
The TPR experiments were performed by dosing $0.4 \mathrm{H}_{2}$ (5.0 purity, Air Liquide) molecules per $\mathrm{MgO}$ surface atom $\left(1 \mathrm{~cm}^{2}=2.25 \times 10^{15}\right.$ surface atoms) followed by $0.4 \mathrm{C}_{2} \mathrm{H}_{4}$ (3.5 purity, Westfalen Germany) ethylene molecules per $\mathrm{MgO}$ surface atom, using a calibrated molecular beam doser at a crystal temperature of $100 \mathrm{~K}$. This molecular beam doser is based on the design of John T. Yates, Jr and provides a superior method for dosing defined amounts of gases in vacuum $[43,44]$. The crystal was then positioned approximately $5 \mathrm{~mm}$ away from a skimmer leading to a differentially pumped QMS (Balzers QMA 430, Liechtenstein) chamber, utilizing electron impact ionization for massselecting product cations. A temperature ramp was then applied (Eurotherm 2408) and the ion signal of $30 \mathrm{~m} / \mathrm{z}$ (ethane parent peak) was recorded. The HD-exchange was performed in the same manner except a gas mixture of $\mathrm{H}_{2}$ and $\mathrm{D}_{2}(100.00 \%$, Westfalen Germany) was used where each partial pressure equaled 0.4 molecules per surface atom. A mass of $3 \mathrm{~m} / \mathrm{z}$ was measured for these experiments. The $D_{2}$ TPDs were also performed with a dosage of 0.4 molecules per surface atom.

The pulsed molecular beam (PMB) technique used in our lab has been described previously $[40,45,46]$ and a brief overview will be given here. A piezo pulser, which allows for highly reproducible gas pulses, was filled with a 2 Torr background pressure of ethylene. From the time resolved QMS response to a single ethylene pulse it was determined that there are $\sim 10^{14}$ gas molecules per pulse giving a local pressure of $\sim 5 \times 10^{-7}$ mbar. A deuterium background pressure of 2 $\times 10^{-6} \mathrm{mbar}$ was established with a leak valve and the temperature of the crystal was increased to 300 K. The crystal was again placed approximately $5 \mathrm{~mm}$ in front of the skimmer (same QMS as for the TPD experiments) and ethylene was pulsed onto the surface at a rate of $0.1 \mathrm{~Hz}$. The ethane production was measured by monitoring the mass signal at $31 \mathrm{~m} / \mathrm{z}$ with an oscilloscope (LeCroy Wave-runner 44Xi-A). Within the signal wave we define a quasi-steady state regime, where the ethane production maintains a constant value over the course of $80 \mathrm{~ms}$. Approximately 20 pulses are then recorded and the quasi steady state signal is averaged and used to calculate the turn over frequency (TOF). The calibration of the mass spectrometer was performed by determining the QMS signal of a saturated monolayer of $\mathrm{CO}$ on $\mathrm{Pt}(111)$ from a TPD experiment and then determining the sensitivity factor between ethane and $\mathrm{CO}$. It should be noted that although we measure ethane at $\mathrm{m} / \mathrm{z}=31$ the factor between the signal from equal amounts of pure ethane $(\mathrm{m} / \mathrm{z}=30)$ and deuterated ethane, measured on the peak $\mathrm{m} / \mathrm{z}=31$, is roughly 2 [47]. As this error is systematic, no influence on 
the trend observed, or even the order of magnitude of the signal, occurs. See refs. $[40,45]$ for more technical details.

Infrared reflection absorption spectroscopy (Thermo Electron Corp. Nicolet FT-6700) was performed in single reflection mode with an external MCT-detector (Thermo Electron Corp., MCTATRS). At the experimental steps indicated in the main paper a background spectra was acquired by averaging 256 scans at a resolution of $4 \mathrm{~cm}^{-1}$ with the sample at $\sim 100 \mathrm{~K} .10$ Langmuir $(\mathrm{L})$ of $\mathrm{CO}$, or $20 \mathrm{~L}$ of ethylene, was dosed and a sample spectra recorded with the same parameters as the background.

Computational Methods To model the $\mathrm{Pt}_{\mathrm{n}} / \mathrm{MgO}$ systems, with $\mathrm{n}=9,10$ and 13 , we employed a four-layer $\mathrm{MgO}(100)$ slab with a calculational cell consisting of $7 \times 6$ unit cells; each layer consisted of $42 \mathrm{Mg}$ and 42 oxygen atoms with the atoms in the bottom layer held stationary (at the experimental lattice constant of $4.21 \AA$ ) and the atoms in the other three layers allowed to relax to the optimal atomic arrangement. The experiments in this paper were performed for $\mathrm{MgO}$ films with thickness of 10 layers or more. Under these conditions there is no effect of the underlying $\mathrm{Mo}(100)$ substrate on the behavior at the top of the film. Indeed the effect of the Mo substrate has been predicted early on theoretically [34] to be limited to MgO films with a thickness of 4-5 layers, (see also reactivity measurements). This has been verified experimentally in later reactivity studies of gold nanoclusters supported on $\mathrm{MgO}$ films of variable thicknesses [32] and most convincingly through work function measurements [48]. The above-mentioned prediction and experimental verifications justify the procedure used in our calculations where no effect of the underlying Mo substrate on the charging behavior at the $\mathrm{MgO} / \mathrm{Pt}$ nanocluster interface is considered.

The optimal (minimum total energy) configurations were determined through unconstrained relaxation of the atomic positions, using a conjugate gradient search with the total energy calculated with the use of first-principles methodologies (density functional theory, DFT) converged within 0.001 $\mathrm{eV}$. The bare clusters were positioned on the $\mathrm{MgO}(100)$ surface, and their configuration, together with the positions of the atoms of the underlying substrate, was optimized via unconstrained relaxations as noted above. The calculational supercell, which included the $\mathrm{MgO}(100)$ slab and a $24 \AA$ thick vacuum region, was periodically replicated. Once the optimal cluster structures have been determined the binding (adsorption) energies and atomic positions of the reactants (that is the individual $\mathrm{H}_{2}$ and $\mathrm{C}_{2} \mathrm{H}_{4}$ molecules, as well as multiply adsorbed, and co-adsorbed, systems) were determined through total 
energy minimization. This relaxation procedure allows for adsorbate/reactant-induced structural relaxations of the underlying metal cluster and metal-oxide $(\mathrm{MgO}(100)$ substrate; this form of adsorbate-induced cluster-catalyst relaxation is a manifestation of a more general class of cluster relaxation processes termed fluxionality [31] which includes also dynamical reaction-induced cluster fluxionality.

In the first principle steered-reaction pathway (SRP) calculations of the reaction profiles (pathways), a reaction coordinate was judiciously chosen; typically, the reaction coordinate consists of the distance between two atoms of the reactant molecules (for example an adsorbed $\mathrm{H}$ atom of a dissociated hydrogen molecule and the $\mathrm{C}$ atom of a reacting ethylene molecule); the reaction coordinate can in general be a combination of geometrical parameters (interatomic distances and/or angles). For each value of the reaction coordinate, the total energy of the system, calculated with density functional theory (DFT) was optimized through unconstrained relaxation of all of the other degrees of freedom of the system (reactants, other adsorbents, Pt cluster atoms and MgO slab atoms atoms). The reaction profiles (reaction paths) were obtained via repeating such calculations for a sequence of values of the chosen reaction coordinate. These calculations yield results that are the same as, or very close to, those obtained by other methods, e.g., the nudged elastic band and variants thereof; see the discussion on pp 89 and 90 in Reference [1].

The first-principles electronic structure calculations used the density functional theory (DFT) method employing the VASP-DFT package, using a plane-wave basis with a kinetic energy cutoff of $400 \mathrm{eV}, \mathrm{PAW}$ pseudopotentials[49] and the PBE generalized gradient approximation (GGA) for the exchange-correlation potential [50-54]. $\Gamma$ - point sampling of the Brillouin zone was used. 


\section{Results and discussion}

\section{$\operatorname{Pt}(111)$}

Initial experiments were performed on a $\mathrm{Pt}(111)$ single crystal in order to reproduce previously reported data and provide a standard for comparison to the results obtained for hydrogenation catalyzed by size-selected clusters deposited on magnesia $(\mathrm{MgO}(100))$. Fig. 1a shows a recorded ethylene hydrogenation TPR curve displaying a peak at $245 \mathrm{~K}$ that correlates well with spectra reported in earlier literature $[55,56]$. IRRAS measurements performed for ethylene adsorbed on the $\mathrm{Pt}(111)$ surface at $100 \mathrm{~K}$ reproduced the well-known $\mathrm{CH}$ stretch of di- $\sigma$ bonded ethylene at $2910 \mathrm{~cm}^{-1}$ (Fig. 1b) and after heating to temperatures above $300 \mathrm{~K}$, the signal from ethylidyne is present at $1339 \mathrm{~cm}^{-1}[57]$.

\section{Size-Selected Clusters:}

\section{One-catalytic-cycle experiments: TPR}

Ethylene hydrogenation TPR measurements were performed on size-selected clusters consisting of 7 to 40 platinum atoms, and their resulting spectra are shown in Fig. 2a. The integrals of the TPR curves (including Pt(111)), normalized to the number of atoms per cluster, are shown in Fig. 2b. The spectra show no generation of ethane for $\mathrm{Pt}_{7-9}$, whereas $\mathrm{Pt}_{10-40}$ all exhibit varying degrees of cluster catalyzed ethane production. The TPR data exhibit two important deviations from the Pt(111) result. First, the integrated intensity per atom clearly shows a size-dependent trend, with a peak at $\mathrm{Pt}_{13}$ and the larger sizes converging to the $\mathrm{Pt}(111)$ single crystal value. Second, the reaction peak temperature measured for the clusters, $150 \mathrm{~K}$ is approximately $90 \mathrm{~K}$ lower than that measured on the Pt(111) surface, indicating a lower activation barrier on the clusters compared to the single crystal surface. In contrast to the classification of the ethylene hydrogenation reaction as structure insensitive, our TPR data indicate that in this cluster size-range a size-dependent reactivity emerges.

In light of the accepted mechanism for ethylene hydrogenation where $\pi$-bonded ethylene is the active species, IRRAS was performed on adsorbed ethylene to explore possible cluster sizedependence of the bonding nature of ethylene to the clusters. Fig. $2 \mathrm{~d}$ shows the comparative spectra measured for $\mathrm{Pt}_{7}, \mathrm{Pt}_{13}$ and $\mathrm{Pt}(111)$. No adsorption band in the $\mathrm{CH}$ stretch region was observed on the clusters, but this is however, not unexpected due to the very low intensity of $\pi$-bonded ethylene [13]. 
The interaction of the clusters with hydrogen was investigated as a possible reason for the reactivity onset. Results of the $\mathrm{HD}$ exchange TPR experiment performed on $\mathrm{Pt}_{7}, \mathrm{Pt}_{8}, \mathrm{Pt}_{9}, \mathrm{Pt}_{10}$ and $\mathrm{Pt}_{13}$, shown in Fig. 3a, clearly indicate that a similar onset effect is found between $\mathrm{Pt}_{9}$ and $\mathrm{Pt}_{10}$ for the $\mathrm{HD}$ exchange, as the aforementioned one measured for the ethylene hydrogenation reaction (see Fig. 2a,b). The possibility that perhaps hydrogen did not dissociate on these smaller cluster sizes, has, however, been discarded due to the otherwise well-known spontaneous dissociation of hydrogen (and deuterium) molecules on platinum. Indeed, our theoretical calculations confirmed that hydrogen does dissociate on all cluster sizes and provided a clear perspective on the HD exchange results (see below).

A deuterium TPD measurement was also carried out before and after the TPR ethylene hydrogenation experiment. The results are displayed in Fig. $3 \mathrm{~b}$ for $\mathrm{Pt}_{9}, \mathrm{Pt}_{10}$ and $\mathrm{Pt}_{13}$; the smaller desorption profile from $\mathrm{Pt}_{13}$ is not unexpected, as for this experiment a lower coverage was used compared to that used for $\mathrm{Pt}_{10}$ and $\mathrm{Pt}_{9}$. The spectra show that before the reaction, all of the cluster sizes manifest a clear desorption peak, measured at 195,220 and $215 \mathrm{~K}$ for $\mathrm{Pt}_{9}, \mathrm{Pt}_{10}$ and $\mathrm{Pt}_{13}$, respectively. After the ethylene hydrogenation TPR experiment, no desorption peak is found from all cluster sizes. From these experiments we rule out the possibility that the onset of ethylene hydrogenation catalytic activity at $\mathrm{Pt}_{10}$ originates from the inability of either ethylene or deuterium to adsorb/react on $\mathrm{Pt}_{9}$; additionally these experiments show that the result of the interaction with ethylene is size independent, that is, on all cluster sizes ethylene causes a blockage of deuterium adsorption sites, likely due to dehydrogenation.

These temperature-programmed and IRRAS investigations show that although the change in reactivity was clear and readily measurable, investigating the underlying cause is more challenging. As has already been mentioned, spectroscopic detection of ethylene on clusters is very unlikely due to the weak $\mathrm{CH}$ stretch signal. As no size-dependent chemical properties could be found to explain the result, we turned to predictions obtained through first-principles theoretical calculations. 


\section{Structures and Charging Effects [40]}

In light of the above observed size-dependencies, it is evident that uncovering the atomicarrangement motifs of the sub-nanometer-size supported Pt cluster catalysts is imperative to advancing our understanding of the physical principles underlying the above-noted apparent structural sensitivity of the catalyzed reactions. The development and implementation of efficient and practical optimization strategies (global optimization in particular) is a vexing problem of great importance in diverse fields, including the physical sciences.

The optimal (lowest-energy) structures of the surface-supported $\mathrm{Pt}_{9}, \mathrm{Pt}_{10}$ and $\mathrm{Pt}_{13}$ clusters determined through first-principles calculations. The structure determination was based on extensive searches, guided by cluster structural motifs gathered for other systems and by previously proposed search methodologies [58]; various initial trial structures were employed, including optimal and isomeric gas-phase structures, and face-capped forms of smaller seed clusters. The results of these searches are displayed in Fig. 4(a-f). It is evident that these clusters prefer a three-dimensional (3D) prismatic two-layer structure with the second layer having a smaller (or the same, e.g. $\mathrm{Pt}_{13}$ ) number of atoms as the first layer; denoting by I and $\mathrm{m}$ the number of atoms in the first (in contact with the $\mathrm{MgO}(100)$ substrate) and second layers and with $\mathrm{k}$ giving the number of capping end-atoms, the structures of $\mathrm{Pt}_{n}, \mathrm{n}=9,10$, and 13, can be designated as $(1, \mathrm{~m})+\mathrm{k}$, namely: $(6,3)$ for $\mathrm{Pt}_{9},(6,3)+1$ for $\mathrm{Pt}_{10}$, and $(6,6)+1$ for $\mathrm{Pt}_{13}$. From the three-dimensionality of these structures, it is apparent that the above-measured structural sensitivity is not associated with a structure dimensionality cross-over for clusters with $n>9$. It is pertinent to recall here that the onset of enhanced activation of $\mathrm{CO}$ oxidation by supported $\mathrm{Au}_{n} / \mathrm{MgO}$ has been attributed originally [3] and corroborated experimentally [59] to be correlated with the appearance of 3D 2-layer cluster for $n \geq 8$. On the other hand, explanation of a similarly measured enhancement of $\mathrm{CO}$ oxidation reaction on $\mathrm{Pt}_{\mathrm{n}} / \mathrm{TiO}_{2}(110)$ by a conjectured dimensionality crossover of the adsorbed $\mathrm{Pt}_{n}$ clusters $[60,61]$ has been refuted in recent DFT calculations $[62,63]$.

Additionally, we observe that these adsorbed clusters possess a large number of higherenergy isomeric structures (with the preferred ones having 3D geometries), reflecting the complex nature of their potential energy surface (see supplementary information to ref. [40]) Although we focus 
in the following on the lowest-energy clusters, it is possible that some higher-energy isomers are also present on the supporting surface.

The unconstrained relaxation procedure employed in these structural investigations (as well as in determination of the reaction pathway) allows for adsorbate/reactant-induced structural relaxations of the underlying metal cluster and metal-oxide $(\mathrm{MgO}(100)$ substrate; this form of adsorbate-induced cluster-catalyst relaxation is a manifestation of a more general class of cluster relaxation processes termed fluxionality [31], which includes also cluster relaxation in the course of a chemical reaction, named dynamical reaction-induced cluster fluxionality, By considering systematically only relaxed configuration, the size-dependent fluxional propensity of clusters in the size regime studied here becomes one of the characteristics that determine the response of the clusters to the presence of reactants and intermediates (e.g. ethylidyne) and the consequent sizedependent reactivities and the course of reactions catalyzed by these clusters. Finally, in addition to being of physical significance in the description of the mechanisms of cluster-catalyzed reactions, the inter-isomer structural conversion shown in Fig. 5 illustrates the use of adsorbate-induced and dynamical reaction-induced cluster fluxionality as a method for uncovering structural isomers; in fact, Fig 5 records the process that led us to discover the ground-state structure of the magnesiasupported $\mathrm{Pt}_{13}$ cluster, $\mathrm{Pt}_{13} / \mathrm{MgO}(100)$ (see also Fig. 4(e,f)).

The spin state of the bare adsorbed Pt clusters is $N_{\uparrow}-N_{\downarrow}=2$ (where $N_{s}$ is the number of electrons with spin $s=\uparrow$ or $\downarrow$ ), and they are found, through total charge differences (calculated before and after adsorption and shown in Fig. $4(\mathrm{a}, \mathrm{c}, \mathrm{e}))$ and Bader charge analysis (BCA, see Fig. 4 (b,d,f)), to be negatively charged with the total excess Bader electronic charges on the adsorbed cluster being: $\delta \rho_{B}\left[\mathrm{Pt}_{n} / \mathrm{MgO}\right]=1.68$ e, 1.78 e and 1.92 e for $n=9,10$ and 13 , respectively. We find that whereas the total excess electronic charges on the $n=9$ and 10 clusters are quite similar, close to a third $(0.51 \mathrm{e})$ of $\delta \rho_{\mathrm{B}}\left[\mathrm{Pt}_{10} / \mathrm{MgO}\right]$ is located for the $\mathrm{Pt}_{10}$ cluster on the capping atom (the 10th atom in Fig. $4 b$ ); similarly, a large contribution to $\delta \rho_{B}\left[\mathrm{Pt}_{13} / \mathrm{MgO}\right]$ is also found on the capping atom (the $1^{\text {st }}$ atom of $\mathrm{Pt}_{13}$, shown in Fig. 4f, which is found to have an excess local charge of $0.34 \mathrm{e}$ out of a total of $1.92 \mathrm{e}$, with the atoms marked 7 having also a large excess charge of 0.36 .. For the $\mathrm{Pt}_{10}$ cluster this excess charge distribution leaves a smaller amount of excess charge $(1.78 \mathrm{e}-0.51 \mathrm{e}=1.27 \mathrm{e})$ to be distributed over the remaining nine atoms of the $\mathrm{Pt}_{10}$ cluster in comparison with the total excess charge (1.68 e) available for distribution over the compact (no capping atom) $\mathrm{Pt}_{9}$ triangular prism 
cluster. This smaller amount of negative excess charge on most of the atoms of the $\mathrm{Pt}_{10}$ cluster compared to the $\mathrm{Pt}_{9}$ case, are found to influence binding sites, adsorption energies, and consequent reaction pathways on the adsorbed clusters as well as demonstrating that, in this size-range, even the addition of a single atom, while not changing the basic geometric structure, can drastically alter the electronic properties of a cluster.

Previous investigations (see review in ref. 11) identified two adsorption modes of ethylene on the (111) surface of platinum, the $\pi$ and di- $\sigma$ modes, where in the former the ethylene is thought to coordinate to a single Pt atom through a $\pi$ bond, with the ethylene maintaining (at least to a large extent) $\mathrm{sp}^{2}$ hybridization, and in the latter the adsorbed molecule is attached to two adjacent Pt atoms (in a $\eta^{2}$ manner) through two $\sigma$ bonds showing a significant extent of $\mathrm{sp}^{3}$ hybridization. On the extended $\mathrm{Pt}(111)$ surface the $\pi$-bonded ethylene was found to be the preferred adsorption configuration only at very low temperatures, whereas for $T=100-240 \mathrm{~K}$ di- $\sigma$ ethylene is preferred under UHV condition, with ethylidyne forming at higher temperatures. All the theoretical computational studies [15] corroborated the experimentally observed preference for di- $\sigma$ over the $\pi$ ethylene absorption mode, whereas the weakly adsorbed $\pi$-bonded species is experimentally believed to be the main reactant during hydrogenation of ethylene [13].

Contrary to extended surfaces, for the magnesia-supported $\mathrm{Pt}_{n}(n \geq 9)$ clusters we find essentially the same propensity for adsorption in the $\pi$ or di- $\sigma$ mode, with the corresponding vertical desorption energies (VDE) of the $\mathrm{C}_{2} \mathrm{H}_{4}$ molecules (coadsorbed with a dissociated $\mathrm{H}_{2}$ molecule in neighboring sites) calculated to be VDE $=1.13 \mathrm{eV}$ and $0.93 \mathrm{eV}$ for the $\pi$ and di- $\sigma$ modes, respectively (see Fig. $4(\mathrm{~g}, \mathrm{~h}))$; note in particular the much higher value of the desorption energy for the $\pi$-bonded molecule compared to the corresponding calculated [ $0.87 \mathrm{eV},[15]]$ and measured [0.41 $\pm 0.10 \mathrm{eV}$, [64]] values on the $\mathrm{Pt}(111)$ surface. Furthermore, the $\pi$-bonded molecule [see Fig. $4 \mathrm{~g}$ for adsorption on $\left.\mathrm{Pt}_{10} / \mathrm{MgO}\right)$ ] has a C-C bond length $d(C-C)=1.425 \AA$ which is close to the experimental value [65] of $1.41 \AA$; this value is somewhat larger than $\mathrm{d}(\mathrm{C}-\mathrm{C})=1.334 \AA$ value found in the isolated $\mathrm{C}_{2} \mathrm{H}_{4}$ molecule, indicating a certain degree of $\mathrm{sp}^{3}$ rehybridization. In the di- $\sigma$ adsorbed ethylene molecule we find evidence for a larger degree of $\mathrm{sp}^{3}$ hybridization, with $\mathrm{d}(\mathrm{C}=\mathrm{C})=1.469 \AA$ which is closer to the value found in the isolated $\mathrm{C}_{2} \mathrm{H}_{6}$ molecule $(\mathrm{d}(\mathrm{C}-\mathrm{C})=1.528 \AA \mathrm{A})$. Similar results were found by us for the two adsorption modes of ethylene on the supported $\mathrm{Pt}_{13}$ cluster (see the $\pi$-bonded adsorption configuration, marked 0 , with $\mathrm{VDE}=1.54 \mathrm{eV}$ in Fig. 8a). 
The charge redistributions for both ethylene adsorption modes reflect the frontier orbital interactions leading to ethylene chemisorption, described by the Dewar-Chatt-Duncanson (DCD) model $[66,67]$, involving electron donation from the highest occupied $(\pi)$ ethylene orbital into an empty d orbital of the metal, and back-donation from the filled Pt d-orbitals into the ethylene lowest unoccupied (antibonding) $\pi^{*}$ orbital. The resulting electron charge isosurfaces (see right panels in Fig. $4 \mathrm{~g}$ and $4 \mathrm{~h}$ ) portray the resulting redistribution of the electron density. Both the above bonding processes, as well as the repulsive interactions between the filled frontier orbitals of ethylene and occupied d-states of the metal catalyst particle (termed as Pauli-[68, 69] or four-electron repulsion [70]) depend on: (i) coordination of atoms making up the Pt particle, with reduced coordination, such as at edge and corner atoms, resulting in decreased repulsion, and (ii) occupation of metal d-states (with the Pauli repulsive interaction between filled orbitals of the metal cluster and the adsorbate increasing at cluster sites having larger excess electron charge). Site-coordination and charging patterns can be ascertained from the calculated information given in Fig. 4(a-f), and similar considerations apply also to the catalyzed ethylene hydrogenation mechanism discussed below. In this context it is pertinent to recall here our systematic study [71] of the effect of charging by cluster-substrate interactions on the adsorption and low-temperature combustion reaction of carbon monoxide and dioxygen, catalyzed by MgO-supported gold cluster of sizes similar to those considered in the current investigation.

\section{Reaction mechanisms}

Prior to elaboration of the ethylene hydrogenation reaction pathways we review first theoretical calculations that we carried out in connection with the thermal desorption and H/D exchange measurements discussed by us earlier (see Fig. 3). These calculations are of particular interest here because the dissociation of molecular hydrogen is a key first step in the HP ethylene hydrogenation mechanism. First we recall in Fig. $6 a$ the $H D$ exchange spectra recorded in the isotopic H/D exchange (scrambling) TPR measurements for $\mathrm{Pt}_{9}, \mathrm{Pt}_{10}$ and $\mathrm{Pt}_{13}$. (see Fig. $3 \mathrm{~b}$ for data measured also for other cluster sizes). These spectra display a limited activity for the $\mathrm{Pt}_{9}$ cluster compared to that of the larger clusters (peaking at $T \sim 200 \mathrm{~K}$ ), along with the measured record for the $\mathrm{Pt}(111)$ surface that shows onset of $\mathrm{H} / \mathrm{D}$ exchange at a higher temperature and a peak centered at $260 \mathrm{~K}$.

Hydrogen molecules are predicted from our DFT calculations to dissociatively adsorb on all Pt atoms of the bottom layer of $\mathrm{Pt}_{9}$ (Fig. 6b), but do not bind to top layer atoms of the cluster; the only 
way that an $\mathrm{H}$ atom may adsorb on the top layer is by diffusing (with an activation energy of $0.65 \mathrm{eV}$ ) from a bottom atom after dissociation, see Fig. $6 b(i i))$. The limited adsorption and dissociation of hydrogen on the $\mathrm{Pt}_{9}$ cluster correlate with the measured $\mathrm{H} / \mathrm{D}$ exchange (Fig. 6a). On the other hand, dissociative $\mathrm{H}_{2}$ adsorption occurs readily at all sites of the bottom layer of the supported $\mathrm{Pt}_{10}$ and $\mathrm{Pt}_{13}$ clusters, with diffusion of the dissociated adsorbed hydrogen atoms between bottom layer sites entailing small barriers of the order of $0.1-0.3 \mathrm{eV}$, and interlayer diffusion requiring barriers that are as low as $0.40 \mathrm{eV}$. Unlike the case of the smallest cluster $\left(\mathrm{Pt}_{9}\right) \mathrm{H}_{2}$ dissociatively adsorbs on one of the second layer atoms of the $\mathrm{Pt}_{10}$ clusters (see Fig.6c(ii), diffusing to a neighboring upper layer atom with an activation energy of $0.66 \mathrm{eV}$ ), and the $\mathrm{Pt}_{13}$ cluster dissociatively adsorbs $\mathrm{H}_{2}$ at all sites (except one, see right arrow, Fig. $6 \mathrm{~d}$ and Supplementary Fig. 1). The abundant sites for dissociative adsorption on both the bottom and top layers of the $\mathrm{Pt}_{10}$ and $\mathrm{Pt}_{13}$ clusters and the facile inter-site $\mathrm{H}$-diffusion on these clusters correlate with the measured low temperature $H / D$ exchange signal (Fig. 6a). It is pertinent to remark here that although we exhibit here (and in the following) results that show a single hydrogen molecule and one ethylene molecule adsorbed (and/or reacting) on a given cluster, we have verified (particularly for the $\mathrm{Pt}_{10}$ and $\mathrm{Pt}_{13}$ clusters) that dissociative adsorption of $\mathrm{H}_{2}$ maintains regardless of the number of $\mathrm{H}_{2}$ molecules adsorbed on the cluster, and that similar adsorption characteristics are obtained when two ethylene molecules are co-adsorbed.

To gain insights into the microscopic ethylene hydrogenation mechanism we carried out extensive first-principles steered reaction-pathway simulations. For $\mathrm{Pt}_{9}$, no low-activation-energy hydrogenation channel was found in the simulations, in agreement with the TPR results (Fig. 2). On the other hand, multiple reaction pathways, characterized by relatively low activation barriers, were found in SRP simulations for the larger clusters, as illustrated in Figs. 7 and 8 for $\mathrm{Pt}_{10}$ and $\mathrm{Pt}_{13}$, respectively.

The simulated pathways starting from a m-bonded ethylene molecule co-adsorbed with a dissociated $\mathrm{H}_{2}$ on the $\mathrm{Pt}_{10}$ and $\mathrm{Pt}_{13}$ clusters are shown in Figs. $7 \mathrm{a}$ and $8 \mathrm{a}$, respectively. For both cluster sizes we observe two successive energy barriers (denoted as $\Delta \mathrm{E}_{\mathrm{T}}(\mathrm{i}), \mathrm{i}=1,2$ ) corresponding to the two hydrogenation steps of the HP mechanism, whose heights are sufficiently low to permit the experimentally observed low temperature reactivity, see Fig. 2 . In the first reaction step (marked (0)-(i) in Fig. $7 \mathrm{a}$ for $\mathrm{Pt}_{10} / \mathrm{MgO}$ ) one of the adsorbed hydrogen atoms approaches the nearest carbon atom (initially $\mathrm{d}\left(\mathrm{C}^{(1)} \mathrm{H}^{(1)}\right)=2.432 \AA$ ). The first activation barrier $\Delta \mathrm{E}_{\mathrm{T}}{ }^{(1)}=0.55 \mathrm{eV}$, is characterized by $\mathrm{d}\left(\mathrm{C}^{(1)-}\right.$ $\left.\mathrm{H}^{(1)}\right)=1.25 \AA$ and $\mathrm{d}\left(\mathrm{C}^{(1)}-\mathrm{C}^{(2)} ; \Delta \mathrm{E}_{\mathrm{T}}^{(1)}\right)=1.487 \AA$ (compare with $\mathrm{d}(\mathrm{C}-\mathrm{C})=1.487 \AA$ calculated for gaseous 
ethyl $\mathrm{C}_{2} \mathrm{H}_{5}$ ) portraying formation of an adsorbed ethyl-like top-of-the-barrier intermediate. In the local minimum (marked (ii)) that follows the first barrier $d\left(C^{(1)}-H^{(1)}\right)=1.105 \AA, d\left(C^{(1)}-C^{(2)} ; i i\right)=1.529 \AA$, and the adsorbed ethyl intermediate is inclined with respect to the cluster $\left[\mathrm{d}\left(\mathrm{C}^{(1)}-\mathrm{Pt}^{(1))}=3.065 \AA\right.\right.$ and $d\left(C^{(2)-}-\mathrm{Pt}^{(1))}=2.080 \AA\right]$. The closer value at the barrier-top of $d\left(C^{(1)}-C^{(2)} ; \Delta E_{T}^{(1)}\right)$ to $d\left(C^{(1)}-C^{(2)} ;\right.$ ii) then to the initial state of the adsorbed ethylene, $\mathrm{d}\left(\mathrm{C}^{(1)}-\mathrm{C}^{(2)} ;(0)\right)=1.425 \AA$, qualifies $\Delta \mathrm{E}_{\mathrm{T}}^{(1)}$ as a "late transition state barrier". Full hydrogenation occurs in the second activation process - (ii) $\mathrm{C}_{2} \mathrm{H}_{5} \rightarrow$ (ii) $\Delta \mathrm{E}_{\mathrm{T}}^{(2)}=$ $0.52 \mathrm{eV} \rightarrow$ (iv) adsorbed $\mathrm{C}_{2} \mathrm{H}_{6}$ with $\mathrm{VDE}=0.1 \mathrm{eV}$ - resulting in the product ethane molecule. The pathways of the hydrogenation reaction of the di- $\sigma$ adsorbed ethylene molecule (shown for $\mathrm{Pt}_{10} / \mathrm{MgO}$ in Fig. $7 b)$ resembles the one described above, with the first activation barrier being lower $(0.33 \mathrm{eV})$. Similar results were found in our SRP simulations for the $\mathrm{Pt}_{13} / \mathrm{MgO}$ cluster (see Fig. $8 \mathrm{a}$ for the $\pi$ bonded ethylene molecule).

The principal result of our analysis to this point is the discovery and explanation of the onset of low-temperature ethylene hydrogenation processes (peaking at temperatures of $\sim 150 \mathrm{~K}$ ) on magnesia-supported 3D $\mathrm{Pt}_{n}$ clusters for $\mathrm{n} \geq 10$, with the calculated activation barriers for both the $\pi$ and di- $\sigma \mathrm{C}_{2} \mathrm{H}_{4}$ adsorption modes on the Pt cluster catalysts being significantly smaller than those obtained for the $\mathrm{Pt}(111)$ surface; compare with values calculated for the lowest coverage (1/9) on $\mathrm{Pt}(111)$ [15]. This reflects significant enhancement of the hydrogenation reaction, achieved through the use of supported platinum clusters of $<1 \mathrm{~nm}$ diameter (on the order of 10-15 Pt atoms); note however the absence of catalytic activity of $3 \mathrm{D} \mathrm{Pt}_{n}$ clusters with $\mathrm{n} \leq 9$. The measured larger hydrogenation reactivity of the $\mathrm{Pt}_{13}$ cluster correlates with the calculated larger number of active sites on this cluster for both $\mathrm{H}_{2}$ dissociative adsorption (see Fig. 6d), and low-barrier hydrogenation of coadsorbed ethylene and hydrogen.

In addition to the low-activation-energy pathways found on the $\mathrm{Pt}_{n} / \mathrm{MgO}(\mathrm{n} \geq 10)$, we have found on these clusters (particularly on the larger cluster, $\mathrm{Pt}_{13} / \mathrm{MgO}$ ) several reaction sites characterized by higher ethylene-hydrogenation activation energy barriers; typically $\Delta \mathrm{E}_{\mathrm{T}}>0.8-1.0 \mathrm{eV}$. Most significantly, along with the low-temperature hydrogenation pathways (Fig. $7(a, b)$ ) we found for the $\mathrm{Pt}_{10} / \mathrm{MgO}$ catalyst a relatively low-barrier dehydrogenation reaction channel resulting in formation of ethylidyne (Fig. 7c), whereas for the larger $\mathrm{Pt}_{13} / \mathrm{MgO}$ cluster we found multiple high-energy ethylidyne formation pathways; see Fig. 8(c,d) manifesting energy-barriers of $\Delta \mathrm{E}_{\mathrm{T}} \sim 1.2-1.3 \mathrm{eV}$. This finding implies for $\mathrm{Pt}_{13}$ an onset of significant ethylidyne formation upon heating to higher temperatures ( $\mathrm{T} \sim$ 
350-400 K), which acts as a blocking, or poisonous, agent for ethylene hydrogenation; for the $\mathrm{Pt}_{10}$ clusters such alternative reaction channels are open already at $300 \mathrm{~K}$, see e.g Fig, 7c.

In all cases (including hydrogenation of $\mathrm{C}_{2} \mathrm{H}_{4}$ on $\mathrm{Pt}(111)$ ) the microscopic reaction mechanism has been found to follow a frontier orbital description that find its origins in an adaptation of the DCD model; here the addition of a hydrogen atom to the adsorbed molecule is described as an agnostic process (a term used to refer specifically to situations in which a hydrogen atom is covalently bonded to both a carbon and a transition metal atom [72]), with the change in the $\mathrm{C}-\mathrm{H}$ distance affecting (increasing) the energy gap between the bonding $\left(\sigma_{\mathrm{CH}}\right)$ and antibonding $\left(\sigma_{\mathrm{CH}}{ }^{*}\right)$ states that shift away from the Fermi level as the $\mathrm{C}-\mathrm{H}$ distance reduces (or equivalently the $\mathrm{Pt}-\mathrm{H}$ distance increases). At the top of the activation barrier, the interaction between the $\sigma_{\mathrm{CH}}$ and $\sigma_{\mathrm{CH}}{ }^{*}$ orbitals with the s-, p- and delectrons of the Pt clusters brings about orbital mixing that may be described in term of the DCD donation and back-donation terms, culminating in attachment of the transferred $\mathrm{H}$ atoms (initially bonded to the Pt cluster) to the adsorbed molecule; for further discussion see ref. [73].

\section{Isothermal multi-catalytic-cycle experiments: PMB}

To go beyond the above one-catalytic-cycle experiments the reactivity of the size-selected $\mathrm{Pt}$ clusters is subsequently studied under isothermal, quasi-steady-state, conditions with the use of a PMB of ethylene. Under such circumstances all the reaction channels that open at temperatures up to the selected temperature of the experiment are operative, including parallel reaction paths that are potentially detrimental to the full hydrogenation of ethylene to ethane, namely the aforementioned coking reactions such as the one resulting in the formation of ethylidyne. Fig. 9a shows the calculated TOF at $300 \mathrm{~K}$ for $\mathrm{Pt}_{8-15} \mathrm{Pt}_{20}, \mathrm{Pt}_{25}, \mathrm{Pt}_{30}$ and $\mathrm{Pt}(111)$. The results show a clear sizedependent activity, with a maximum for $\mathrm{Pt}_{13}$. The aforementioned reactivity onset effect (when increasing the cluster size from $\mathrm{Pt}_{9}$ to $\mathrm{Pt}_{10}$ ) is absent, as both clusters have a similar activity under isothermal conditions at $300 \mathrm{~K}$ (similar to that measured on the extended Pt(111) surface). The sizedependent behavior observed here is direct evidence that the ethylene hydrogenation is actually a structure sensitive reaction in the sub-nanometer particle size regime. The similar activity measured for the $\mathrm{Pt}_{8-10}$ clusters is also not unexpected, as exhibiting the same TOF as $\mathrm{Pt}(111)$ merely means that these cluster sizes do not exhibit the characteristics required for structure sensitivity, i.e. there are cluster sizes which are structure insensitive. This demonstrates the power that atomic resolution has 
in revealing size-dependent properties of particles, whereas a polydisperse sample might not show that there is any particle size effect present.

Compared to a TPR measurement (where the temperature is increased linearly), the PMB isothermal experiments represent a fundamentally different physical system, as multiple reaction pathways can be available for ethylene on the clusters, e.g. ethylidyne formation. This indicates that while the clusters in their bare state exhibited an onset effect, (when increasing the cluster size from $\mathrm{Pt}_{9}$ to $\mathrm{Pt}_{10}$ ) the property leading to the drastic difference in reactivity becomes non operative under isothermal conditions at an increased temperature.

Included in Fig. 9a are the measured TOFs of the reaction performed at $300 \mathrm{~K}$ after the sample was heated to $400 \mathrm{~K}$ and exposed to ten ethylene pulses in the same deuterium background pressure. The increased temperature has now caused a deactivation of the more active sizes $\left(\mathrm{Pt}_{11}\right.$, $\mathrm{Pt}_{12}, \mathrm{Pt}_{13}, \mathrm{Pt}_{14}$ and $\left.\mathrm{Pt}_{15}\right)$, but the larger sizes $\left(\mathrm{Pt}_{20}, \mathrm{Pt}_{25}\right.$ and $\left.\mathrm{Pt}_{30}\right)$ show little to no deactivation. The fact that $\mathrm{Pt}_{8-10}$ have also not deactivated indicates that cluster sintering is not the cause of the observed deactivation, as these sizes should also show an appreciable decrease in activity. Rather, we conclude that the fact that the smaller cluster sizes do not deactivate indicates that the temperature increase to $400 \mathrm{~K}$ has initiated a similar process (on $\mathrm{Pt}_{11}, \mathrm{Pt}_{12}, \mathrm{Pt}_{13}, \mathrm{Pt}_{14}$ and $\mathrm{Pt}_{15}$ ) to what occurred on the smaller sizes already at $300 \mathrm{~K}$, as now these sizes all exhibit structure insensitivity.

It is well known that strongly adsorbed carbon species form on platinum single crystals and particles at $300 \mathrm{~K}$. Indeed, the formation of carbonaceous species has been proposed as the reason behind the observed structure sensitivity $[10,20,74]$. Our data show that there is strong evidence that certain Pt cluster sizes show a resistance to the formation of these species. This intrinsic resistance can, however, be overcome by increasing the reaction temperature; note however, that even at $400 \mathrm{~K} \mathrm{Pt}_{20}$ exhibits a resistance to passivation, indicating that each cluster size must be individually evaluated for this behavior.

In a previous publication we calculated (using DFT) the $d$-band center $\left(\varepsilon_{c}^{\mathrm{M}}\right)$ for model $\mathrm{M}_{30}(\mathrm{M}$ $=\mathrm{Ni}, \mathrm{Pd}, \mathrm{Pt}$ ) clusters in order to establish a trend across group VIII metal nanoparticles[46]. We were then able to account for the observed difference in deactivation during ethylene hydrogenation by using a model [73] whereby the position of $\varepsilon_{\mathrm{c}}^{\mathrm{M}}$ can be used to predict the activation barrier for ethylene dehydrogenation or hydrogenation, with a $\varepsilon_{\mathrm{c}}^{\mathrm{M}}$ closer to the Fermi level resulting in a lowered barrier for dehydrogenation. The calculated $\varepsilon_{\mathrm{c}}^{\mathrm{M}}$ values were $-1.33,-1.86$ and $-2.40 \mathrm{eV}$ for $\mathrm{Ni}, \mathrm{Pd}$ and $\mathrm{Pt}$, 
respectively $[46,65]$. The observed trend in the hydrogenation/dehydrogenation behavior followed the systematic variation of the $\varepsilon_{\mathrm{c}}^{\mathrm{M}}$ for the different metals, whereby Ni showed the lowest hydrogenation activity and greatest proclivity to deactivate, whereas Pt exhibited the highest activity and strongest resistance to deactivation. $\mathrm{Pt}(111)$ has a calculated $\varepsilon_{\mathrm{c}}^{\mathrm{M}}$ of $-2.25 \mathrm{eV}$ [75] which leads to a prediction of lower activity/facile deactivation compared to $\mathrm{Pt}_{30}$, which is in agreement with the findings displayed in Fig. 9a. Interestingly, we find that even though the diameter of $\mathrm{Pt}_{30}$ is only of the order of $1 \mathrm{~nm}$, the calculated value of its $\varepsilon_{\mathrm{c}}^{\mathrm{M}}(-2.40 \mathrm{eV})$ shows an approach to the $\mathrm{Pt}(111)$ bulk value of $-2.25 \mathrm{eV}$, resulting (with further increase in particle size) in the emergence of structure insensitivity even for particles of nanometer size diameters.

Because of the lack of direct spectroscopic data from ethylene adsorbed on size-selected clusters, an indirect approach was employed where $\mathrm{CO}$ was used as a chemical probe. It is well known that carbon species on Pt particles can cause a red-shift in the $\mathrm{CO}$ stretch frequency. Figure 9b shows representative IR spectra from $\mathrm{Pt}_{9}, \mathrm{Pt}_{10}, \mathrm{Pt}_{13}, \mathrm{Pt}_{20}, \mathrm{Pt}_{30}$ and $\mathrm{Pt}(111)$ after pulsing at $300 \mathrm{~K}$ and after the deactivation step and subsequent activity measurement again at $300 \mathrm{~K}$ have been performed. The raw spectra all show a similar trend: decrease in intensity and stronger red shift as the temperature has been increased. A summary of the $\mathrm{CO}$ stretch position, including the measured value from the clean clusters, is given in Fig. 9c. Here, it is evident that the bare clusters show a redshift as the particle size decreases, as previously observed [76, 77]. After the reaction at $300 \mathrm{~K}$, a red-shift is observed for all the cluster sizes and the single crystal. $\mathrm{Pt}_{9}, \mathrm{Pt}_{10}$ and $\mathrm{Pt}_{13}$ all show a shift of approximately $13 \mathrm{~cm}^{-1}$, while on $\mathrm{Pt}_{20}$ the value increases to $19 \mathrm{~cm}^{-1}$ and on $\mathrm{Pt}_{30}$ the value is $40 \mathrm{~cm}^{-1}$. On $\mathrm{Pt}(111)$ the red-shift is $83 \mathrm{~cm}^{-1}$, similar to the value of $75 \mathrm{~cm}^{-1}$ measured with sum-frequency generation (SFG) on $\mathrm{Pt}(111)$ [78]. The small redshift observed on the smaller cluster sizes could possibly be caused by a change in cluster geometry during the temperature increase, however, in light of the deuterium desorption experiment before and after the TPR measurement, a complete loss of adsorption sites can be rationalized only with co-adsorbed carbon species. The fact that $\mathrm{CO}$ can adsorb and deuterium cannot is also not unknown, as ethylidyne on Pt exhibits a low saturation coverage which can be penetrated by $\mathrm{CO}[76,78,79]$. The much more pronounced red-shift observed from $\mathrm{Pt}_{30}$ indicates a tendency towards extended, $\mathrm{Pt}(111)$, surface behavior.

After the heating experiment to $400 \mathrm{~K}$, the absence of a $\mathrm{CO}$ peak from the data measured for $\mathrm{Pt}_{9}$ indicates that formation of carbon species causes an effective site blocking of $\mathrm{CO}$. As the TOF 
has not changed, the change in the carbon species has not caused an effective impenetrable overlayer to be formed. Indeed the absence of a CO peak does not preclude adsorption, as the very low coverage of clusters means that the concentration may be below the detection limit.

The results from $\mathrm{Pt}_{13}$ and $\mathrm{Pt}_{20}$ both demonstrate that the presence of co-adsorbed carbon species, evidenced in IR after the $300 \mathrm{~K}$ step, does not preclude an enhanced activity (seen for $\mathrm{Pt}_{13}$ ), and that a further redshift after the $400 \mathrm{~K}$ step (seen for $\mathrm{Pt}_{20}$ ) also does not necessarily result in a deactivation, like the one observed for $\mathrm{Pt}_{13}$ making the reaction structure insensitive. This points to a size-dependent carbon chemistry on clusters, where not only the presence of carbon, but also its stoichiometry, bonding geometry, adsorption site and concentration plays a role in the observed activity for ethylene hydrogenation. Determining the nature of these carbon species presents a future challenge. 


\section{Conclusions}

A number of important aspects pertaining to cluster catalysis, structure sensitivity/insensitivity and ethylene hydrogenation emerged as a result of the combined experimental and theoretical investigations described in this paper. First, and foremost, we conclude that classifying a reaction as structure sensitive or insensitive must be reexamined and reevaluated for particle sizes in the nonscalable sub-nanometer regime, where surface chemistry becomes strongly dependent on the precise number of atoms in the catalyzing particle. The combined TPR and PMB investigation has shown that ethylene hydrogenation is indeed a structure sensitive reaction in the sub-nanometer particle size regime of $\mathrm{Pt}_{7}-\mathrm{Pt}_{40}$, with a maximum reactivity observed for $\mathrm{Pt}_{13}$. Structure insensitive behavior was also observed to be inherent for specific cluster sizes at room temperature, and can also be induced in more active sizes (e.g. $\mathrm{Pt}_{13}$ ) by an increase in reaction temperature, which opens up dehydrogenation reaction pathways leading to the formation of carbon species on the clusters. These carbon species were evidenced using IR spectroscopy and were predicted by first-principles calculations. Additionally, there are also sizes (e.g. $\mathrm{Pt}_{20}$ ) where this induced structure insensitive behavior at higher temperature is attenuated, and where extended surface quantities such as the $d$ band center start to become operative $\left(\mathrm{Pt}_{30}\right)$. These results highlight the pitfalls encountered in applying the structure sensitive/insensitive classification (anchored in early investigations carried on larger aggregates) to the analysis of particles in the non-scalable size regime. These results also show that the selectivity and activity of hydrogenation/dehydrogenation reactions can be controlled by utilizing catalyst particles in this size range.

The above findings demonstrate that element specific generalizations of catalytic properties, based on single crystals and nanoparticles, are insufficient in the sub-nanometer size range. The diverse physical and chemical properties demonstrated by this class of materials was also shown, in first-principles simulations, to be operative for similar geometric catalyst structures. Consequently, reactions must be individually tested in an atom-by-atom, size-selective, manner, in order to disentangle and reveal the true catalytic properties of such systems. The application of structure sensitivity or insensitivity criteria served as a most useful concept for the description and systematization of catalytic processes on catalysts comprised of well-defined single crystal facets, i.e. single crystals and nanoparticles synthesized using chemical techniques. However, as the particle size decreases into the regime where essentially all atoms are surface atoms, extrapolations from 
systems of larger size are likely to become inadequate and require instead an atom-by-atom reassessment.

In closing, the results reported here demonstrate that hydrogenation and dehydrogenation reactions can be controlled and tuned by controlling the precise atomic size of the catalyst particle. This has implications for a broad range of chemical processes ranging from chemical synthesis, to food chemistry, and energy applications, where the insight from investigations in the non-scalable size-regime could facilitate a deeper understanding of applied reaction systems, as well as revealing design strategies for catalysts with enhanced activity and/or selectivity.

The influence and reach of John T. Yates, Jr. was ever-present during our investigations. His work on clarifying the mechanism of ethylene hydrogenation, initiating the research field of sizeselected clusters deposited on surfaces in UHV and the technical advances he made in vacuum technology exemplify his broad, continuing scientific impact.

\section{Acknowledgements}

The experimental work has been supported by the European Research Council (ERC) through the advanced research grant (246645-ASC3) and the DFG Project (He3454/23-1). B.Y. was supported by the Air Force Office for Scientific Research (AFOSR) and U.L. by grant No. FG05-86ER45234 from the Office of Basic Energy Sciences of the US Department of energy (DOE). Computations were carried out at the Georgia Tech Center for Computational Materials science. 


\section{References}

[1] U. Heiz, U. Landman, Nanocatalysis, in: Nanoscience and Technology, Springer Berlin Heidelberg, Berlin, Heidelberg, 2007.

[2] U. Heiz, A. Sanchez, S. Abbet, W.-D. Schneider, Catalytic Oxidation of Carbon Monoxide on Monodispersed Platinum Clusters: Each Atom Counts, J. Am. Chem. Soc., 121 (1999) 3214-3217.

[3] A. Sanchez, S. Abbet, U. Heiz, W.-D. Schneider, H. Häkkinen, R.N. Barnett, U. Landman, When Gold Is Not Noble: Nanoscale Gold Catalysts, J. Phys. Chem. A, 103 (1999) 9573-9578.

[4] Y. Watanabe, Atomically precise cluster catalysis towards quantum controlled catalysts, Science and Technology of Advanced Materials, 15 (2014) 063501.

[5] M. Boudart, Catalysis by Supported Metals*, in: H.P.a.P.B.W. D.D. Eley (Ed.) Adv. Catal., Academic Press, 1969, pp. 153--166.

[6] M. Boudart, A. Aldag, J.E. Benson, N.A. Dougharty, C. Girvin Harkins, On the specific activity of platinum catalysts, J. Catal., 6 (1966) 92--99.

[7] M. Boudart, A.W. Aldag, L.D. Ptak, J.E. Benson, On the selectivity of platinum catalysts, J. Catal., 11 (1968) 35--45.

[8] M. Che, C.O. Bennett, The Influence of Particle Size on the Catalytic Properties of Supported Metals, in: H.P.a.P.B.W. D.D. Eley (Ed.) Adv. Catal., Academic Press, 1989, pp. 55--172.

[9] G.C. Bond, The origins of particle size effects in heterogeneous catalysis, Surf. Sci., 156, Part 2 (1985) 966--981.

[10] G.C. Bond, Supported metal catalysts: some unsolved problems, Chem. Soc. Rev., 20 (1991) 441--475.

[11] G.A. Somorjai, K. McCrea, Roadmap for catalysis science in the 21st century: a personal view of building the future on past and present accomplishments, Appl. Catal. A, 222 (2001) 3--18.

[12] H.S. Taylor, A Theory of the Catalytic Surface, Proc. R. Soc. London, Ser. A, 108 (1925) 105--111.

[13] P.S. Cremer, X. Su, Y.R. Shen, G.A. Somorjai, Ethylene Hydrogenation on Pt(111) Monitored in Situ at High Pressures Using Sum Frequency Generation, J. Am. Chem. Soc., 118 (1996) 2942.

[14] J. Horiuti, M. Polanyi, Mechanism of the Ethylene Hydrogenation, Trans. Faraday Soc., 30 (1934) 1164.

[15] Z.-J. Zhao, L.V. Moskaleva, H.A. Aleksandrov, D. Basaran, N. Rösch, Ethylidyne Formation from Ethylene over Pt(111): A Mechanistic Study from First-Principle Calculations, J. Phys. Chem. C, 114 (2010) 12190.

[16] M.K. Ko, H. Frei, Millisecond FT-IR Spectroscopy of Surface Intermediates of C2H4 Hydrogenation over Pt/Al2O3 Catalyst under Reaction Conditions, J. Phys. Chem. B, 108 (2004) 1805.

[17] W. Wasylenko, H. Frei, Direct observation of surface ethyl to ethane conversion over Pt/AlOx cat, J. Phys. Chem. B, 109 (2005) 16873.

[18] T.P. Beebe, J.T. Yates, An in situ infrared spectroscopic investigation of the role of ethylidyne in the ethylene hydrogenation reaction on palladium/alumina, J. Am. Chem. Soc., 108 (1986) 663--671.

[19] M. Boudart, Heterogeneous Catalysis by Metals, J. Mol. Catal., 30 (1985) 27-38.

[20] F. Zaera, Key unanswered questions about the mechanism of olefin hydrogenation catalysis by transition-metal surfaces: a surface-science perspective, Phys. Chem. Chem. Phys., 15 (2013) 11988--12003.

[21] F. Gao, D.W. Goodman, Model Catalysts: Simulating the Complexities of Heterogeneous Catalysts, Annual Review of Physical Chemistry, 63 (2012) 265--286. 
[22] T.P. St.Clair, D.W. Goodman, Metal nanoclusters supported on metal oxide thin films: bridging the materials gap, Topics in Catalysis, 13 (2000) 5--19.

[23] A. Masson, B. Bellamy, Y.H. Romdhane, M. Che, H. Roulet, G. Dufour, Intrinsic size effect of platinum particles supported on plasma-grown amorphous alumina in the hydrogenation of ethylene, Surf. Sci., 173 (1986) 479--497.

[24] A. Sapi, C. Thompson, H. Wang, W.D. Michalak, W.T. Ralston, S. Alayoglu, G.A. Somorjai, Recovery of $\mathrm{Pt}$ Surfaces for Ethylene Hydrogenation-Based Active Site Determination, Catal. Lett., 144 (2014) 1151--1158.

[25] A.M. Doyle, S.K. Shaikhutdinov, S.D. Jackson, H.-J. Freund, Hydrogenation on Metal Surfaces: Why are Nanoparticles More Active than Single Crystals?, Angew. Chem. Int. Ed., 42 (2003) 5240--5243.

[26] A. Binder, M. Seipenbusch, M. Muhler, G. Kasper, Kinetics and particle size effects in ethene hydrogenation over supported palladium catalysts at atmospheric pressure, Journal of Catalysis, 268 (2009) 150--155.

[27] S. Shaikhutdinov, M. Heemeier, M. Bäumer, T. Lear, D. Lennon, R.J. Oldman, S.D. Jackson, H.-J. Freund, Structure--Reactivity Relationships on Supported Metal Model Catalysts: Adsorption and Reaction of Ethene and Hydrogen on Pd/Al2O3/NiAl(110), J. Catal., 200 (2001) 330--339.

[28] W. Ludwig, A. Savara, S. Schauermann, H.-J. Freund, Role of Low-Coordinated Surface Sites in Olefin Hydrogenation: A Molecular Beam Study on Pd Nanoparticles and $\operatorname{Pd}(111)$, ChemPhysChem, 11 (2010) 2319--2322.

[29] U. Landman, B. Yoon, C. Zhang, U. Heiz, M. Arenz, Factors in gold nanocatalysis: oxidation of CO in the non-scalable size regime, Top. Catal., 44 (2007) 145--158.

[30] U. Heiz, R. Sherwood, D.M. Cox, A. Kaldor, J.T. Yates, CO Chemisorption on Monodispersed Platinum Clusters on SiO2: Detection of $\mathrm{CO}$ Chemisorption on Single Platinum Atoms, J. Phys. Chem., 99 (1995) 8730--8735.

[31] H. Häkkinen, S. Abbet, A. Sanchez, U. Heiz, U. Landman, Structural, Electronic, and Impurity-Doping Effects in Nanoscale Chemistry: Supported Gold Nanoclusters, Angew. Chem. Int. Ed., 42 (2003) 1297-1300.

[32] C. Harding, V. Habibpour, S. Kunz, A.N.-S. Farnbacher, U. Heiz, B. Yoon, U. Landman, Control and Manipulation of Gold Nanocatalysis: Effects of Metal Oxide Support Thickness and Composition, J. Am. Chem. Soc., 131 (2009) 538--548.

[33] M.D. Kane, F.S. Roberts, S.L. Anderson, Effects of Alumina Thickness on CO Oxidation Activity over Pd20/Alumina/Re(0001): Correlated Effects of Alumina Electronic Properties and Pd20 Geometry on Activity, J. Phys. Chem. C, 119 (2015) 1359--1375.

[34] D. Ricci, A. Bongiorno, G. Pacchioni, U. Landman, Bonding Trends and Dimensionality Crossover of Gold Nanoclusters on Metal-Supported MgO Thin Films, Physical Review Letters, 97 (2006) 036106.

[35] C. Zhang, B. Yoon, U. Landman, Predicted Oxidation of CO Catalyzed by Au Nanoclusters on a Thin Defect-Free MgO Film Supported on a Mo(100) Surface, J. Am. Chem. Soc., 129 (2007) 2228--2229.

[36] U. Heiz, U. Landman, Nanocatalysis, in, Springer, 2008.

[37] H. Falsig, B. Hvolbæk, I.S. Kristensen, T. Jiang, T. Bligaard, C.H. Christensen, J.K. Nørskov, Trends in the Catalytic CO Oxidation Activity of Nanoparticles, Angew. Chem. Int. Ed., 47 (2008) 4835--4839.

[38] B. Hvolbæk, T.V.W. Janssens, B.S. Clausen, H. Falsig, C.H. Christensen, J.K. Nørskov, Catalytic activity of Au nanoparticles, Nano Today, 2 (2007) 14--18.

[39] T.V.W. Janssens, B.S. Clausen, B. Hvolbæk, H. Falsig, C.H. Christensen, T. Bligaard, J.K. Nørskov, Insights into the reactivity of supported Au nanoparticles: combining theory and experiments, Top. Catal., 44 (2007) 15--26.

[40] A.S. Crampton, Rötzer M. D., Schweinberger F.F.S., Yoon B., Heiz, U., Landman U. , Structure sensitivity in the nonscalable regime explored via catalysed ethylene hydrogenation on supported platinum nanoclusters, Nat. Commun., 7:10389 (2016).

[41] U. Heiz, F. Vanolli, L. Trento, W.-D. Schneider, Chemical Reactivity of size-selected supported clusters: An experimental setup, Rev. Sci. Inst., 68 (1997) 1986-1994. 
[42] F.F. Schweinberger, A.S. Crampton, T. Zimmermann, G. Kwon, C.J. Ridge, S. Günther, U. Heiz, Submonolayer sensitive adsorption study of trichloroethene on single crystal surfaces by means of MIES, UPS and TPD, Surf. Sci., 609 (2013) 18--29.

[43] M.J. Bozack, L. Muehlhoff, J. J. N. Russel, W.J. Choyke, J.T. Yates, Methods in semiconductor surface chemistry, J. Vac. Sci. Tech. A., 5 (1987) 1-8.

[44] A. Winkler, J.T. Yates, Capillary dosing and angular desorption distribution measurements, J. Vac. Sci. Tech. A., 6 (1988) 2929.

[45] C.J. Harding, S. Kunz, V. Habibpour, V. Teslenko, M. Arenz, U. Heiz, Dual pulsed-beam controlled mole fraction studies of the catalytic oxidation of $\mathrm{CO}$ on supported $\mathrm{Pd}$ nanocatalysts, J. Catal., 255 (2008) 234--240.

[46] A.S. Crampton, M.D. Rötzer, F.F. Schweinberger, B. Yoon, U. Landman, U. Heiz, Ethylene hydrogenation on supported $\mathrm{Ni}, \mathrm{Pd}$ and $\mathrm{Pt}$ nanoparticles: Catalyst activity, deactivation and the d-band model, J. Catal., 333 (2016) 51-58.

[47] Y. Amenomiya, R.F. Pottie, Mass spectra of some deuterated ethanes. I. The effect of ionizing voltage, Can. J. Chem., 46 (1968) 1735--1739.

[48] M.E. Vaida, T.M. Bernhardt, C. Barth, F. Esch, U. Heiz, U. Landman, Ultrathin magnesia films as support for molecules and metal clusters: Tuning reactivity by thickness and composition, Phys. Status Solidi B, 247 (2010) 1001--1015.

[49] G. Kresse, D. Joubert, From ultrasoft pseudopotentials to the projector augmentedwave method, Phys. Rev. B, 59 (1999) 1758--1775.

[50] Erratum: Tight bound and convexity constraint on the exchange-correlation-energy functionalsin the low-density limit, and other formal tests of generalized-gradient approximations [Phys. Rev. B \textbackslashtextbf $\backslash 48 \backslash, 11638$ (1993)], Phys. Rev. B, 55 (1997) 13321--13321.

[51] M. Levy, J.P. Perdew, Tight bound and convexity constraint on the exchangecorrelation-energy functional in the low-density limit, and other formal tests of generalizedgradient approximations, Phys. Rev. B, 48 (1993) 11638--11645.

[52] J.P. Perdew, Unified Theory of Exchange and Correlation Beyond Local Density Approximation, in: P. Ziesche, H. Eschrig (Eds.), Akademie Verlag, Berlin, 1991, pp. 11-20.

[53] J.P. Perdew, K. Burke, M. Ernzerhof, Generalized Gradient Approximation Made Simple, Phys. Rev. Lett., 77 (1996) 3865--3868.

[54] J.P. Perdew, J.A. Chevary, S.H. Vosko, K.A. Jackson, M.R. Pederson, D.J. Singh, C. Fiolhais, Atoms, molecules, solids, and surfaces: Applications of the generalized gradient approximation for exchange and correlation, Phys. Rev. B, 46 (1992) 6671--6687.

[55] P. Berlowitz, C. Megiris, J.B. Butt, H.H. Kung, Temperature-programmed desorption study of ethylene on a clean, a hydrogen-covered, and an oxygen-covered platinum(111) surface, Langmuir, 1 (1985) 206.

[56] F. Zaera, On the Mechanism for the Hydrogenation of Olefins on Transition-Metal Surfaces: The Chemistry of Ethylene on Pt(111)†, Langmuir, 12 (1996) 88.

[57] S.B. Mohsin, M. Trenary, H.J. Robota, Infrared identification of the low-temperature forms of ethylene adsorbed on platinum/alumina, J. Phys. Chem., 92 (1988) 5229.

[58] M.D. Wolf, U. Landman, Genetic Algorithms for Structural Cluster Optimization, J. Phys. Chem. A, 102 (1998) 6129-6137.

[59] A.A. Herzing, C.J. Kiely, A.F. Carley, P. Landon, G.J. Hutchings, Identification of Active Gold Nanoclusters on Iron Oxide Supports for CO Oxidation, Science, 321 (2008) 1331-1335.

[60] N. Isomura, X. Wu, Y. Watanabe, Atomic-resolution imaging of size-selected platinum clusters on TiO2(110) surfaces, J. Chem. Phys., 131 (2009) 164707.

[61] Y. Watanabe, X. Wu, H. Hirata, N. Isomura, Size-dependent catalytic activity and geometries of size-selected Pt clusters on TiO2(110) surfaces, Catal. Sci. Tech., 1 (2011) 1490.

[62] S.C. Ammal, A. Heyden, Nature of Ptn/TiO2(110) Interface under Water-Gas Shift Reaction Conditions: A Constrained ab Initio Thermodynamics Study, J. Phys. Chem. C, 115 (2011) 19246--19259. 
[63] D.-e. Jiang, S.H. Overbury, S. Dai, Structures and Energetics of Pt Clusters on TiO2: Interplay between Metal-Metal Bonds and Metal-Oxygen Bonds, J. Phys. Chem. C, 116 (2012) 21880--21885.

[64] J. Kubota, S. Ichihara, J.N. Kondo, K. Domen, C. Hirose, m-bonded ethene on Pt(111) surface studied by IRAS, Surf. Sci., 357-358 (1996) 634-638.

[65] A. Cassuto, M. Mane, J. Jupille, Ethylene monolayer and multilayer on $\mathrm{Pt}(111)$ below 52 K: determination of bond lengths by near-edge X-ray fine structure, Surf. Sci., 249 (1991) 814.

[66] J. Chatt, L.A. Duncanson, Olefin co-ordination compounds. Part III. Infra-red spectra and structure: attempted preparation of acetylene complexes, J. Chem. Soc., (1953) 29392947.

[67] M. Dewar, Bull. Soc. Chim., 18 (1951) C79.

[68] M. Neurock, R.A. van Santen, A First Principles Analysis of C-H Bond Formation in Ethylene Hydrogenation, J. Phys. Chem. B, 104 (2000) 11127-11145.

[69] R.A. van Santen, M. Neurock, Molecular Heterogeneous Catalysis: A Conceptual and Computational Approach, Wiley-VCH, Weinheim, 2006.

[70] P. Sautet, J.-F. Paul, Low temperature adsorption of ethylene and butadiene on

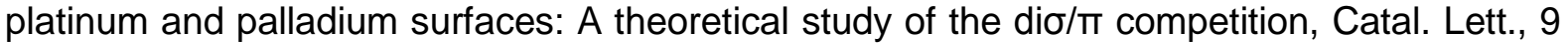
(1991) 245-260.

[71] B. Yoon, U. Landman, A.S. Woerz, J.-M. Antonietti, S. Abbet, K. Judai, U. Heiz, Probing of charging and reactivity of supported gold nanoclusters: Bonding of $\mathrm{CO}$ to gold octamers adsorbed on magnesia, Science, 307 (2005) 403.

[72] M. Brookhart, M.L.H. Green, Carbon-hydrogen-transition metal bonds, J. Organometall. Chem., 250 (1983) 395--408.

[73] V. Pallassana, M. Neurock, Electronic Factors Governing Ethylene Hydrogenation and Dehydrogenation Activity of Pseudomorphic PdML/Re(0001), PdML/Ru(0001), $\mathrm{Pd}(111)$, and PdML/Au(111) Surfaces, J. Catal., 191 (2000) 301-317.

[74] M. Boudart, Heterogeneous catalysis by metals, J. Mol. Catal., 30 (1985) 27--38.

[75] A. Ruban, B. Hammer, P. Stoltze, H.L. Skriver, J.K. Nørskov, Surface electronic structure and reactivity of transition and noble metals, J. Mol. Catal. A, 115 (1997) 421--429.

[76] R.M. Rioux, J.D. Hoefelmeyer, M. Grass, H. Song, K. Niesz, P. Yang, G.A. Somorjai, Adsorption and Co-adsorption of Ethylene and Carbon Monoxide on Silica-Supported Monodisperse Pt Nanoparticles: Volumetric Adsorption and Infrared Spectroscopy Studies, Langmuir, 24 (2008) 198--207.

[77] M.J. Lundwall, S.M. McClure, D.W. Goodman, Probing Terrace and Step Sites on Pt Nanoparticles Using CO and Ethylene, J. Phys. Chem. C, 114 (2010) 7904.

[78] P. Chen, K.Y. Kung, Y.R. Shen, G.A. Somorjai, Sum frequency generation spectroscopic study of CO/ethylene coadsorption on the $\mathrm{Pt}\left(\begin{array}{lll}1 & 1 & 1\end{array}\right)$ surface and $\mathrm{CO}$ poisoning of catalytic ethylene hydrogenation, Surf. Sci., 494 (2001) 289--297.

[79] U. Starke, A. Barbieri, N. Materer, M.A. Van Hove, G.A. Somorjai, Ethylidyne on $\mathrm{Pt}(111)$ : Determination of adsorption site, substrate relaxation and coverage by automated tensor LEED, Surf. Sci., 286 (1993) 1--14. 

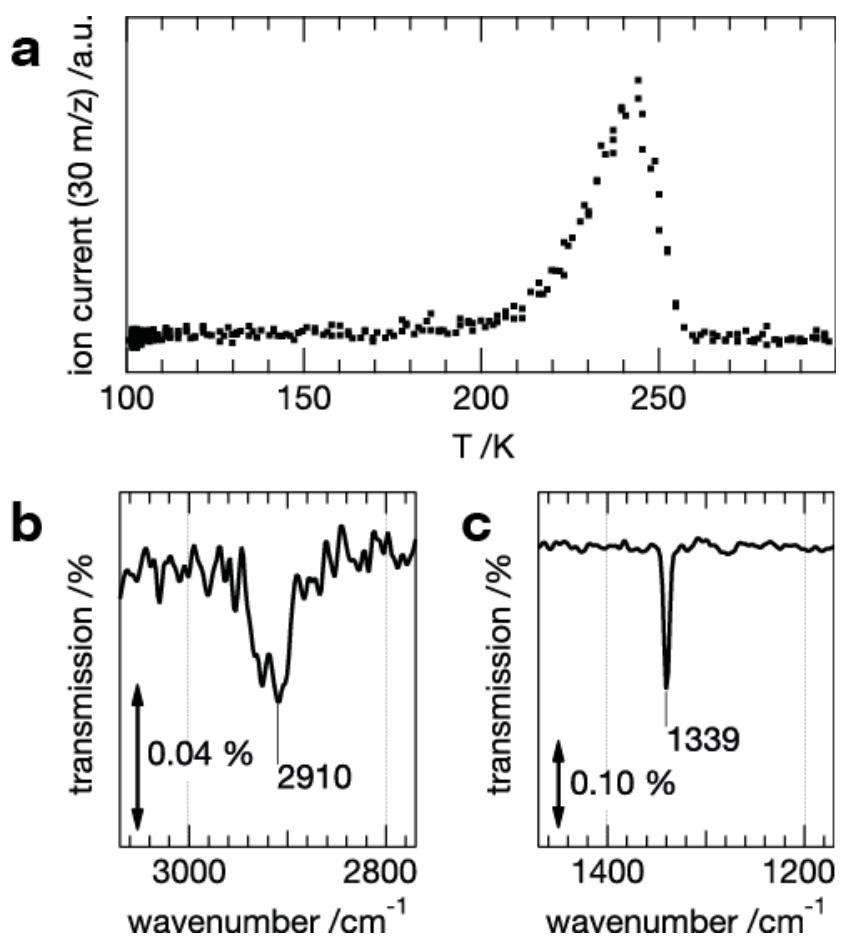

Fig. 1. TPR and IR results from $\mathrm{Pt}(\mathbf{1 1 1})$. (a) ethylene hydrogenation TPR from a $\mathrm{Pt}(111)$ single crystal showing an ethane desorption peak at $245 \mathrm{~K}$. (b) IRRAS spectrum showing the C-H stretch from di- $\sigma$ bonded ethylene at $2910 \mathrm{~cm}^{-1}$ on $\mathrm{Pt}(111)$. (c) IRRAS spectrum showing the $\mathrm{CH}_{3}$ deformation absorption from ethylidyne after heating an ethylene pre-covered $\mathrm{Pt}(111)$ surface to $>300 \mathrm{~K}$. 
a
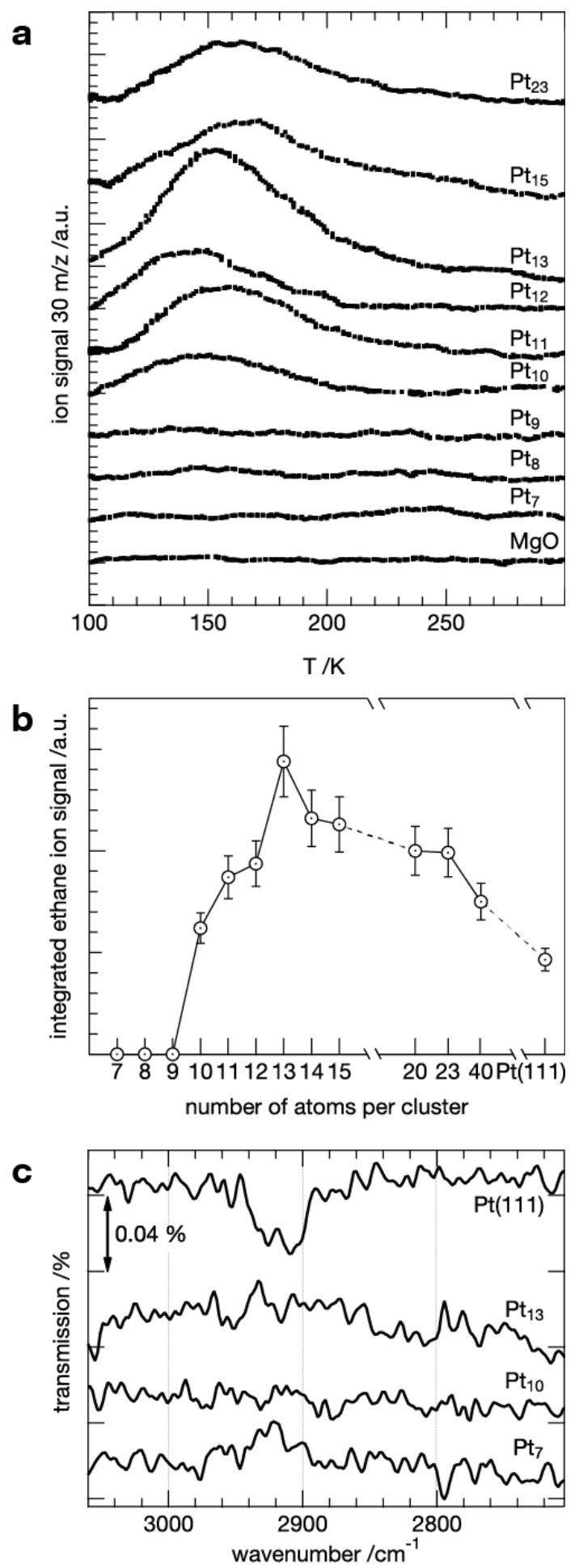

Fig. 2. Cluster TPR and IR Results. TPR from a selection of clusters (a), with their respective integrals normalized to the number of atoms per cluster (b), including the Pt(111) TPR seen in Fig. 1a. Also depicted in (c) are the IRRAS spectra at $100 \mathrm{~K}$ showing the $\mathrm{CH}$ stretch region of ethylene, from size-selected clusters where no $\mathrm{CH}$ stretch can be observed compared to $\mathrm{Pt}(111)$. 
a

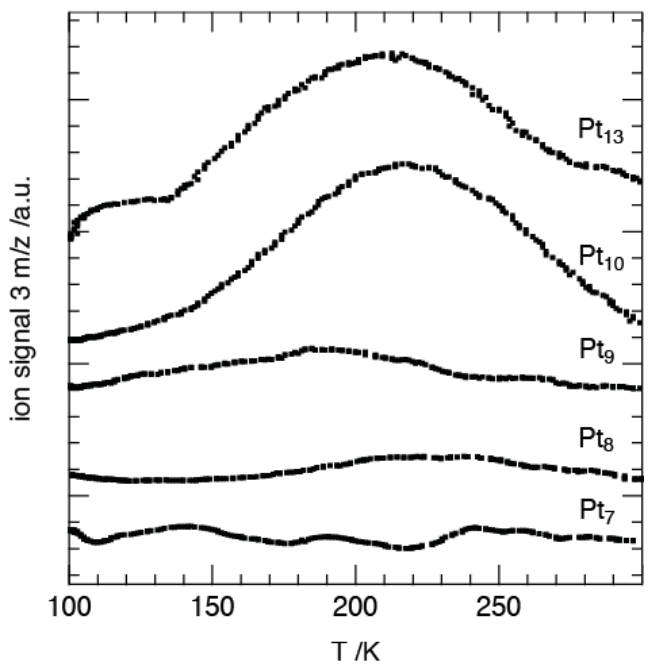

b

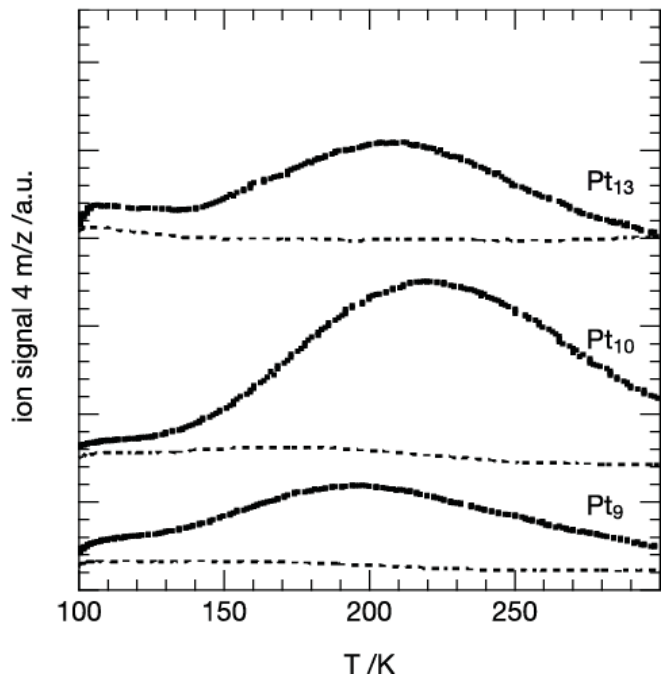

Fig. 3. $D_{2}$ TPD and HD exchange TPR on size-selected clusters. Displayed in (a) are the HD exchange TPR spectra recorded for $\mathrm{Pt}_{7}-\mathrm{Pt}_{10}$ and $\mathrm{Pt}_{13}$. (b) depicts the $\mathrm{D}_{2}$ TPD spectra recorded before (dotted line) and after (dashed line) an ethylene hydrogenation TPR measurement on $\mathrm{Pt}_{9}, \mathrm{Pt}_{10}$ and $\mathrm{Pt}_{13}$. 

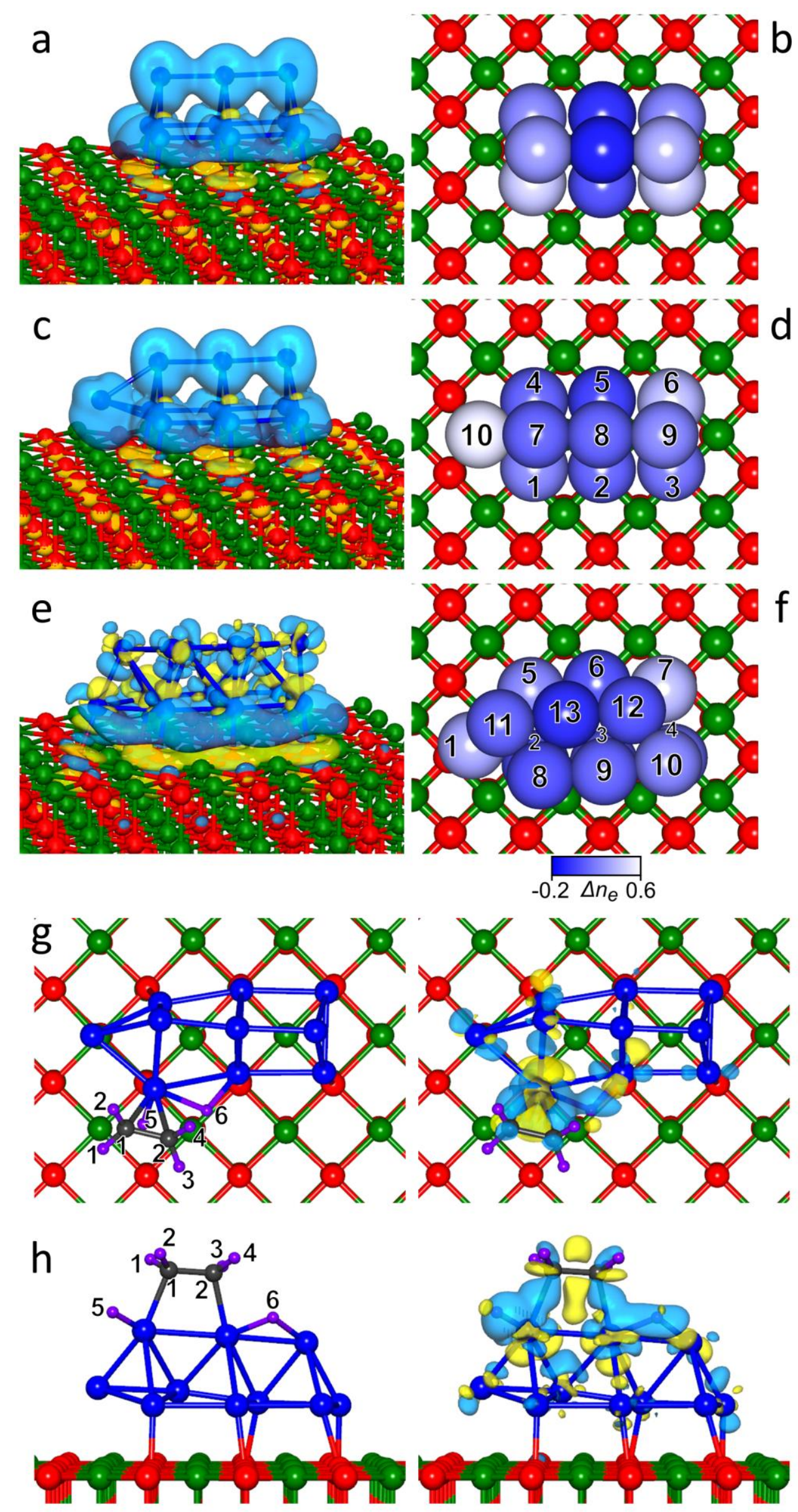
Fig. 4. Optimal adsorption configurations and charge distribution on $\mathbf{M g O}$-supported $\mathbf{P t}_{\mathbf{n}}$ clusters $(n=9,10,13)$. In a, c, and e, the blue and yellow contour hyper-surfaces correspond to excess (light blue) and deficient (yellow) charge distributions obtained as the difference between the total charges before and after adsorption of the clusters; these hypersurfaces are drawn such that the excess electronic (negative) charge inside the light blue hypersurface is $50 \%$ of the total electronic charge and the same for the positive charge inside the yellow hypersurfaces. Bader charge analysis is given in b, $d$, and $f$, with lighter color corresponding to excess number of electrons (that is excess negative charge on the corresponding atom). Coadsorption of $\mathrm{C}_{2} \mathrm{H}_{4}$ and $\mathrm{H}_{2}$ on $\mathrm{Pt}_{10} / \mathrm{MgO}$ is shown in (g) for the ethylene $\pi$-bonding mode to the cluster, and in (h) for the di- $\sigma$ (h) bonding mode. In $\mathrm{g}$ and $\mathrm{h}$ the adsorption geometries are shown on the left, and on the right we depict the bonding frontier orbitals of the adsorption system (the light blue and yellow denote different signs of the wave function); the $\sigma$-type Pt-C bonds are clearly seen in $\mathrm{h}$ (note the directed wave function contours on the right). In both $\mathrm{g}$ and $\mathrm{h}$ atoms 5 and 6 are the proximal dissociated coadsorbed $\mathrm{H}$ atoms. Adapted with permission from ref. [40]. 

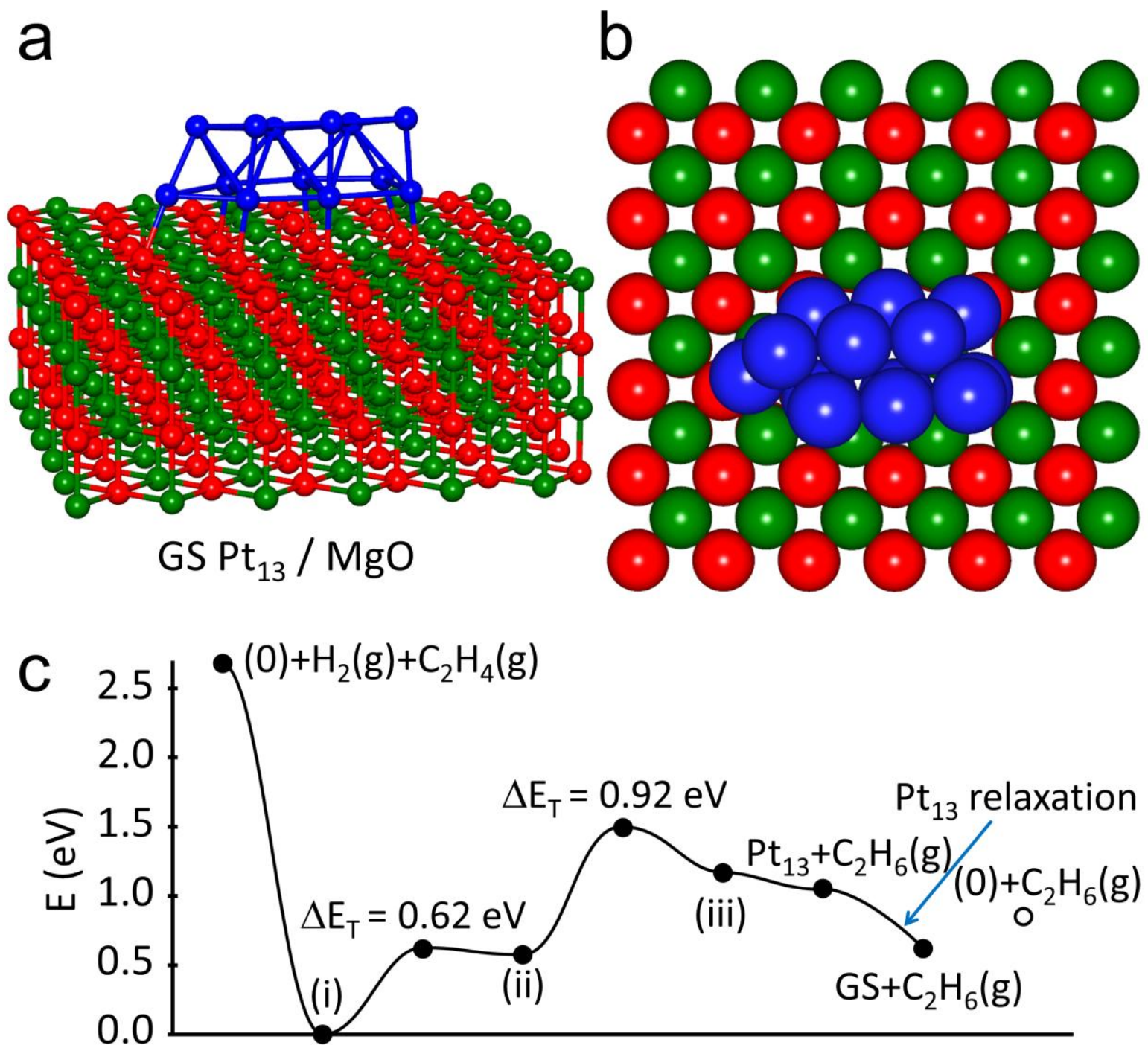

(0)

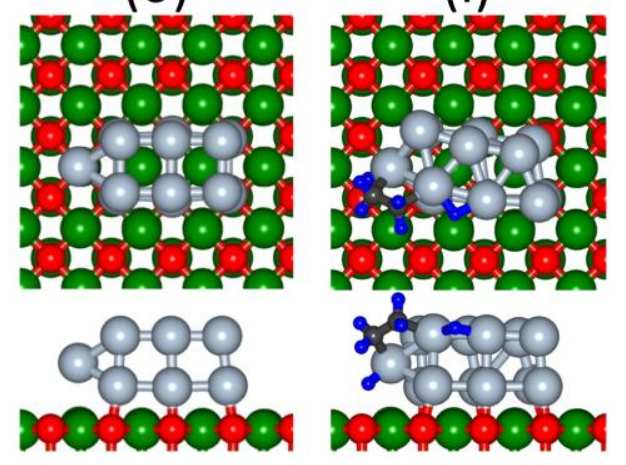

(ii)

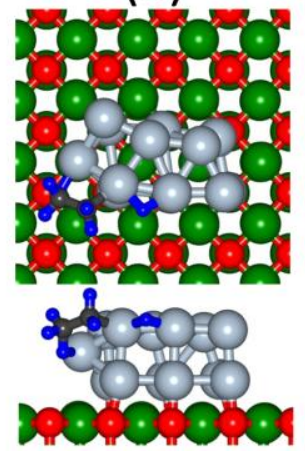

(iii)

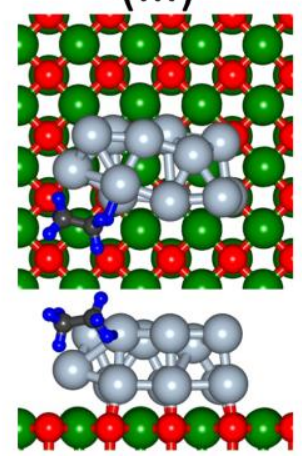

Fig. 5. Adsorption and reaction induced structural fluxionality. (a,b) side (a) and top (b) views of the ground state (GS) structure of $\mathrm{Pt}_{13} / \mathrm{MgO}(100)$. (c) Fluxional transformation from an initial configuration (0) having a higher-in-energy isomer structure with total energy $\Delta \mathrm{E}=0.31 \mathrm{eV}$ above the energy of the ground stateisomer displayed in a and b.. This transformation is induced by the coadsorption of $\mathrm{C}_{2} \mathrm{H}_{4}$ and dissociated $\mathrm{H}_{2}$, see configuration (i). Overcoming the two activation barriers $(0.66 \mathrm{eV}$ and $0.92 \mathrm{eV})$, the product at the end of the ethane $\left(\mathrm{C}_{2} \mathrm{H}_{6}(\mathrm{~g})\right)$-producing hydrogenation 
reaction contains a magnesia-supported $\mathrm{Pt}_{13}$ cluster with the $\mathrm{GS}$ structure (see $\mathrm{a}$ and $\mathrm{b}$ above) with a total energy lowering of the fully relaxed $\mathrm{Pt}_{13} / \mathrm{MgO}$ system energy of $0.31 \mathrm{eV}$ (compare the energy of the states $\mathrm{GS}+\mathrm{C}_{2} \mathrm{H}_{6}(\mathrm{~g})$ denoted by the rightmost filled dot in the energy pathway in (c) with that of the state marked by the rightmost open dot, corresponding to $(0)+\mathrm{C}_{2} \mathrm{H}_{6}(\mathrm{~g})$, where (0) is the bare initial higher-in-energy isomer shown on the left of the bottom row of (c). Adapted with permission from ref. [40]. 


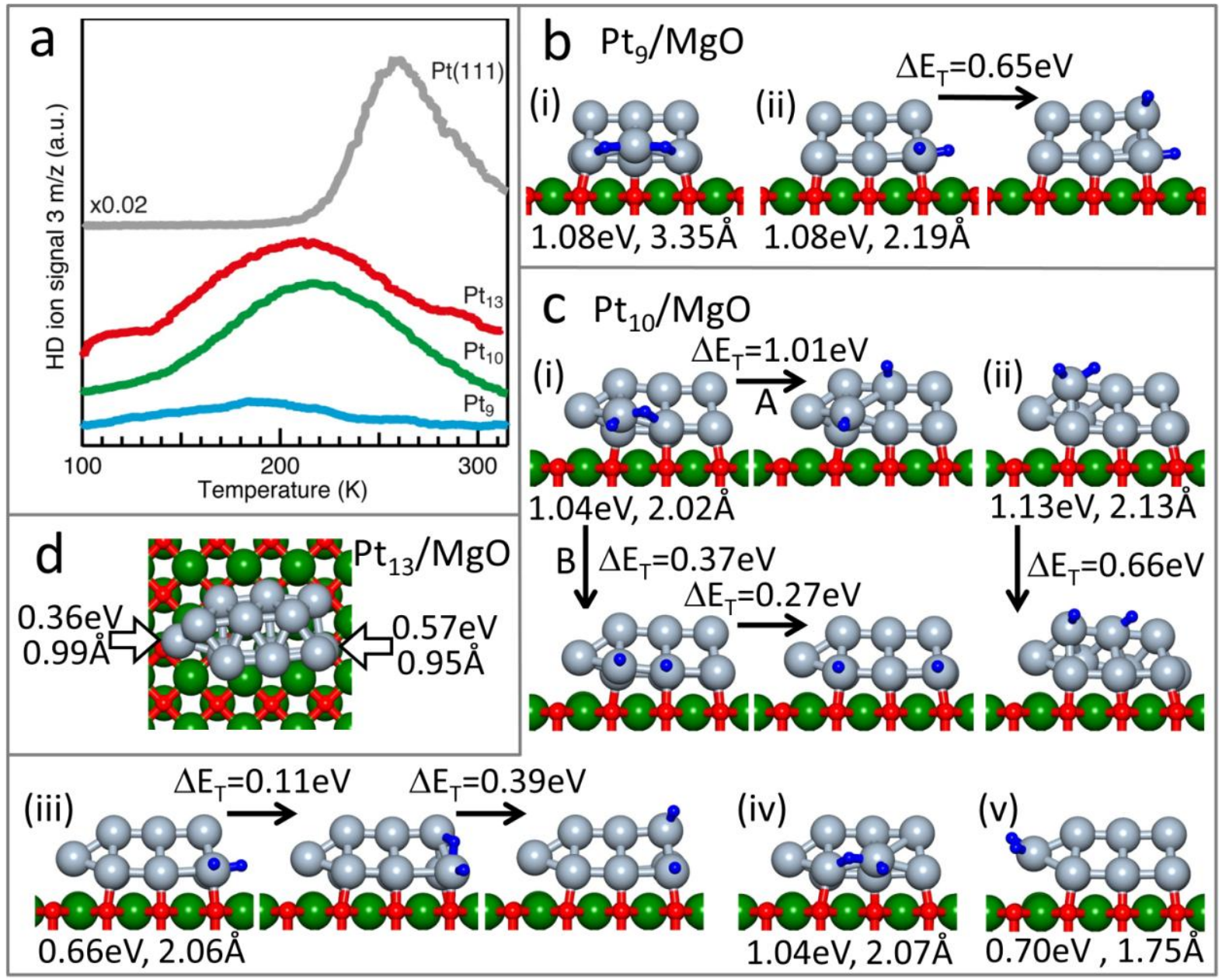

Fig. 6. Hydrogen adsorption on supported platinum clusters. (a) TPR measurements of the H/D exchange on $\mathrm{Pt}_{n} / \mathrm{MgO}, \mathrm{n}=9,10,13$ and on $\mathrm{Pt}(111)$. (b,c) calculated hydrogen dissociative adsorption at various sites, and diffusion on $\mathrm{Pt}_{9} / \mathrm{MgO}$ and $\mathrm{Pt}_{10} / \mathrm{MgO}$, respectively. The pair of values under an adsorption configuration give the $\mathrm{H}_{2}$ adsorption energy and distance between the dissociated $\mathrm{H}$ atoms. $\Delta \mathrm{E}_{\mathrm{T}}$ denote the activation barrier for diffusion between the corresponding $\mathrm{H}$ atom configurations on the Pt cluster. (d) On $\mathrm{Pt}_{13} / \mathrm{MgO} \mathrm{H}_{2}$ dissociates at all sites except the ones marked by an arrow. Spheres colred green denote $\mathrm{Mg}$ atoms, oxygen atoms are colored red, $\mathrm{Pt}$ atoms in gray, and hydrogen atoms are depicted by small blue spheres. The cluster configurations correspond to the lowest energy cluster-adsorption configuration on $\mathrm{MgO}(100)$. Adapted with permission from ref. [40]. 

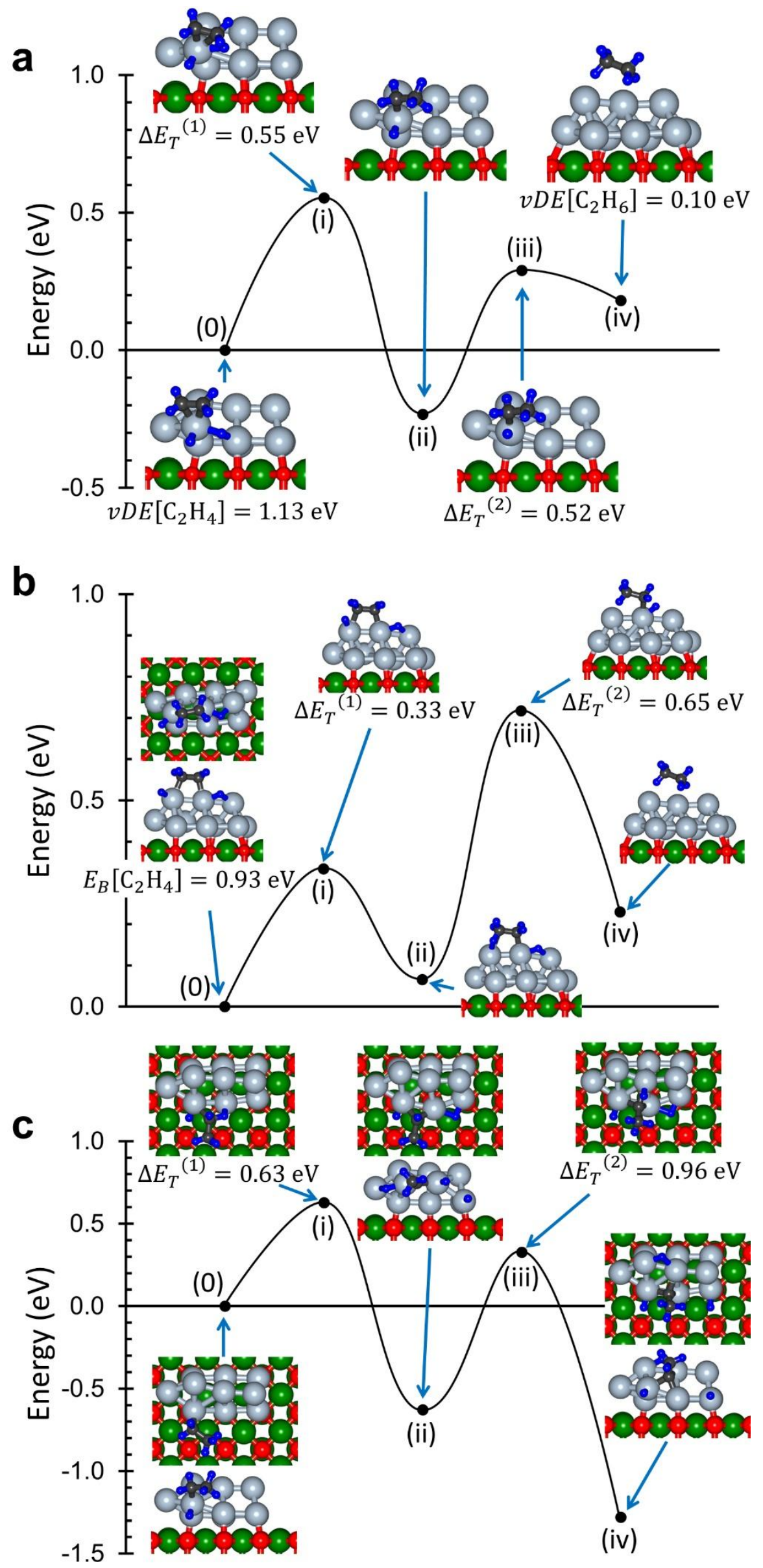
Fig. 7. Calculated first-principles steered reaction pathways (SRP) catalyzed by $\mathbf{P t}_{10} / \mathbf{M g O}$. $(a, b)$ Low-activation-barrier reaction pathways for the $\pi$ (a) and di- $\sigma$ (b) bonded ethylene (configuration on the left, marked 0), coadsorbed with dissociated hydrogen. The activation energy barriers are denoted as $\Delta \mathrm{E}_{\mathrm{T}}^{(\mathrm{k})}, \mathrm{k}=1,2$. The reaction proceeds through the two successive hydrogenation steps described in the Horiuti-Polanyi mechanism. (c) SRP for the low-temperature generation of ethylidyne $\left(\equiv \mathrm{CCH}_{3}\right)$ on $\mathrm{Pt}_{10} / \mathrm{MgO}$, starting from the ethyl $\left(-\mathrm{CH}_{2} \mathrm{CH}_{3}\right)$ intermediate (configuration (0), on the left) generated in the first step of the reaction for the $\pi$-bonded ethylene (depicted as configuration (ii) in the top (a) panel). The two activation barriers correspond to dehydrogenation processes, resulting in a strongly adsorbed ethylidyne molecule $\left(\equiv \mathrm{CCH}_{3}\right.$, configuration (iv)). Surmounting the barrier for the second dehydrogenation process is assisted by the highly exothermic $(\sim 1.35 \mathrm{eV})$ formation of the $-\mathrm{CHCH}_{3}$ intermediate (iii) resulting from the first dehydrogenation step. Adapted with permission from ref. [40]. 

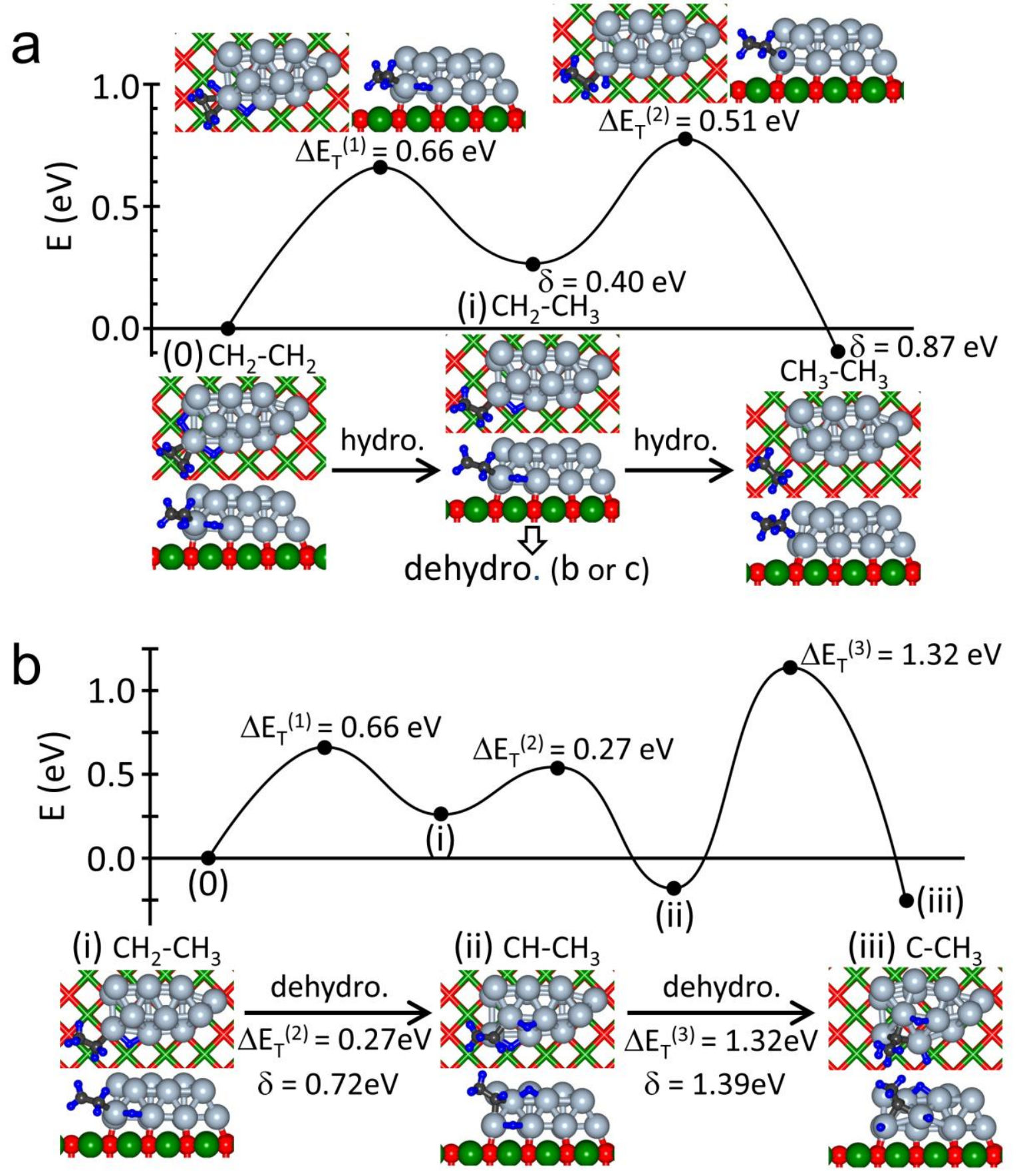

(iii) $\mathrm{C}-\mathrm{CH}_{3}$

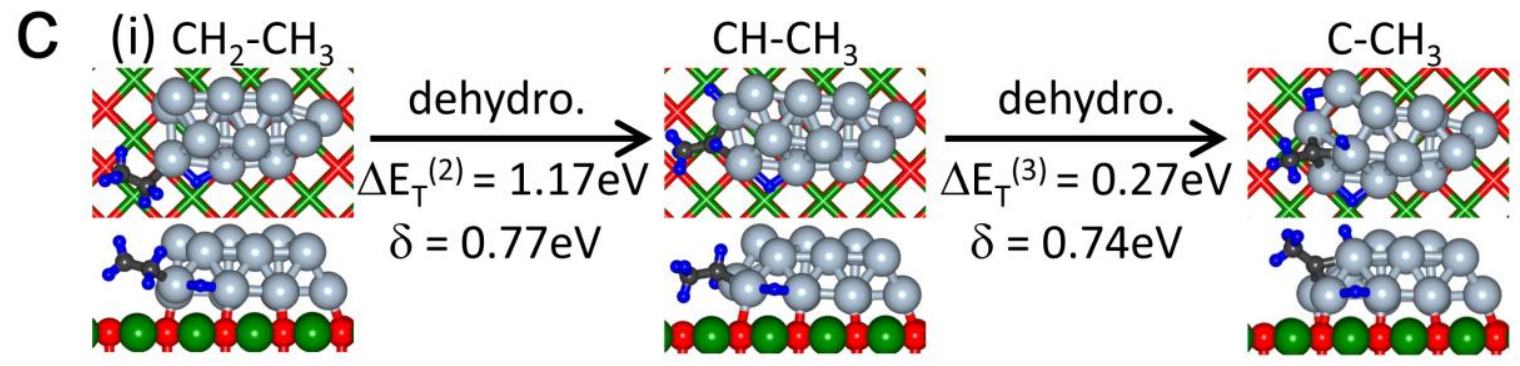

Fig. 8. Calculated first-principles steered reaction pathways (SRP) catalyzed by $\mathrm{Pt}_{13} / \mathbf{M g O}$. (a) Low-activation-barrier reaction pathway for hydrogenation of $\pi$-bonded ethylene configuration coadsorbed with dissociated hydrogen on $\mathrm{Pt}_{13} / \mathrm{MgO}$. The reaction proceeds through the two successive hydrogenation steps described in the HP mechanism. The blue downward arrow near 
configuration (i) indicated the adsorbed ethyl intermediate (i) is the starting configuration for the $\mathrm{B}$ \& $\mathrm{C}$ pathways. (b,c) Alternative reaction channels yielding ethylidyne $\left(\equiv \mathrm{C}-\mathrm{CH}_{3}\right)$, starting from the $\pi-$ bonded ethylene configuration coadsorbed with dissociated hydrogen (marked $(0)$ in a). The ethylene hydrogenation reaction producing ethane (see pathways in a) entails step (0) $\rightarrow$ (i), with $\Delta \mathrm{E}_{\mathrm{T}}{ }^{(1)}=0.66$ $\mathrm{eV}$, followed by the activated second hydrogenation $\left(\Delta \mathrm{E}_{\mathrm{T}}{ }^{(2)}=0.51 \mathrm{eV}\right)$ that yields a weakly adsorbed $\mathrm{C}_{2} \mathrm{H}_{6}$ molecule. Two alternative dehydrogenation reaction channels that lead to formation of ethylidyne (starting from the ethyl, $\mathrm{CH}_{2} \mathrm{CH}_{3}$, intermediate, marked (i) in $\mathrm{A}$, see blue downward arrow in a), are shown in $b$ and $c$. The $b$ and $c$ pathways entail higher energy-barriers $(1.32 \mathrm{eV}$ and $1.17 \mathrm{eV}$, in $\mathrm{b}$ and c, respectively) and thus their activation requires heating above room temperature, resulting in marked poisoning of the ethane-producing hydrogenation channel (see Fig. 5). The graph (profile) of the reaction path is shown only for channel $b$. The energy difference between the top of an activation barrier and the subsequent local minimum on the reaction pathway is given (in $\mathrm{eV}$ ) by $\delta$; this energy gives the height of the reverse reaction step, or it can assist (in part) passage over the subsequent energy barrier. Adapted with permission from ref. [40]. 


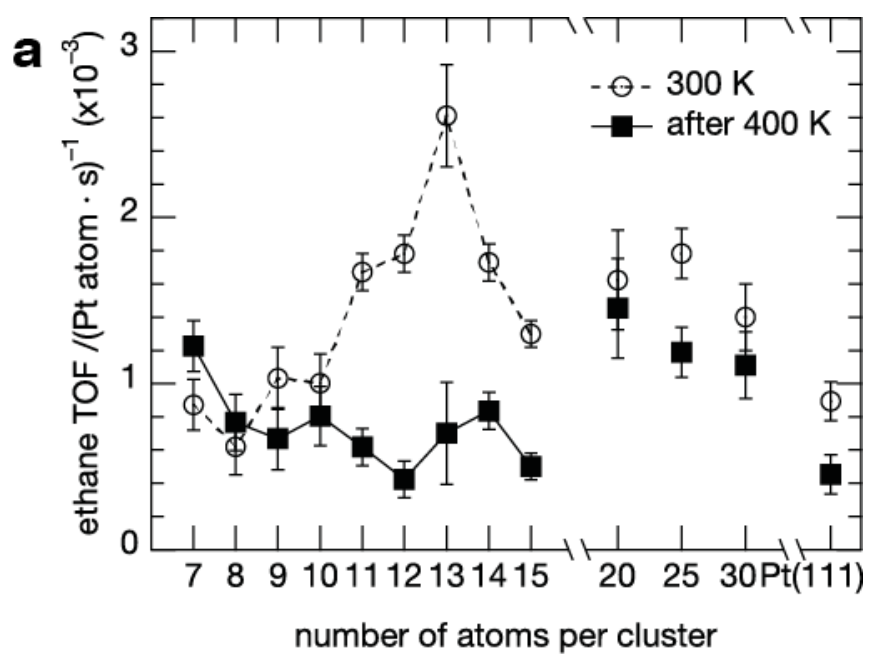

b
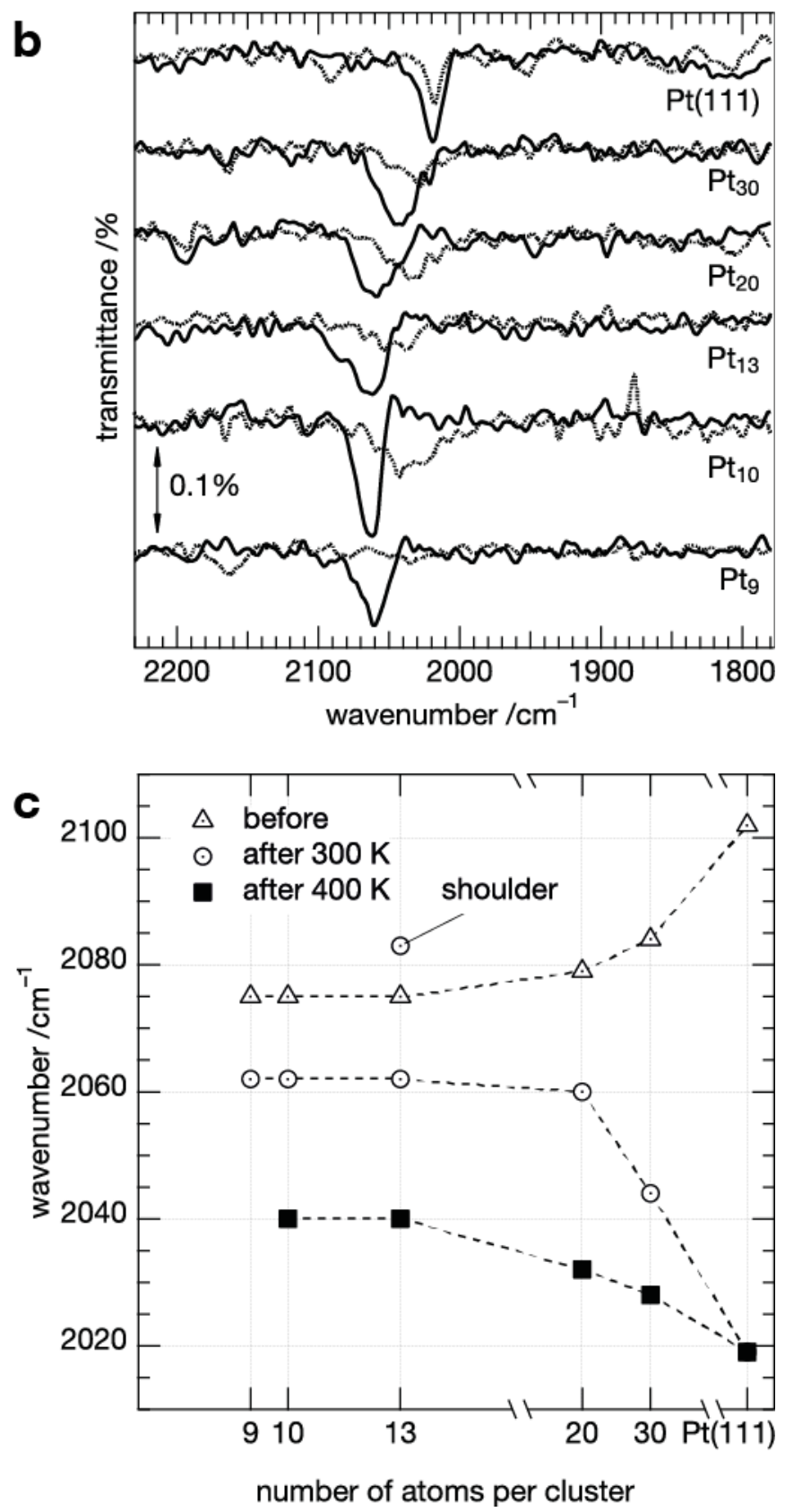
Fig. 9. TOF of Pt Clusters and CO stretch position after reaction steps. (a) depicts the TOF for $\mathrm{Pt}_{7}-\mathrm{Pt}_{30}$ and $\mathrm{Pt}(111)$ (open circles) at $300 \mathrm{~K}$. Included also is the TOF at $300 \mathrm{~K}$ after a (heating) temperature step to $400 \mathrm{~K}$ has been performed (filled squares). (b) displays the IRRAS spectra from the cluster catalysts and $\mathrm{Pt}(111)$ after pulsing at $300 \mathrm{~K}$ and after the temperature step to $400 \mathrm{~K}$, and (c) shows the position of the CO stretch from (b), as well as the position of the clean clusters and $\operatorname{Pt}(111)$. 

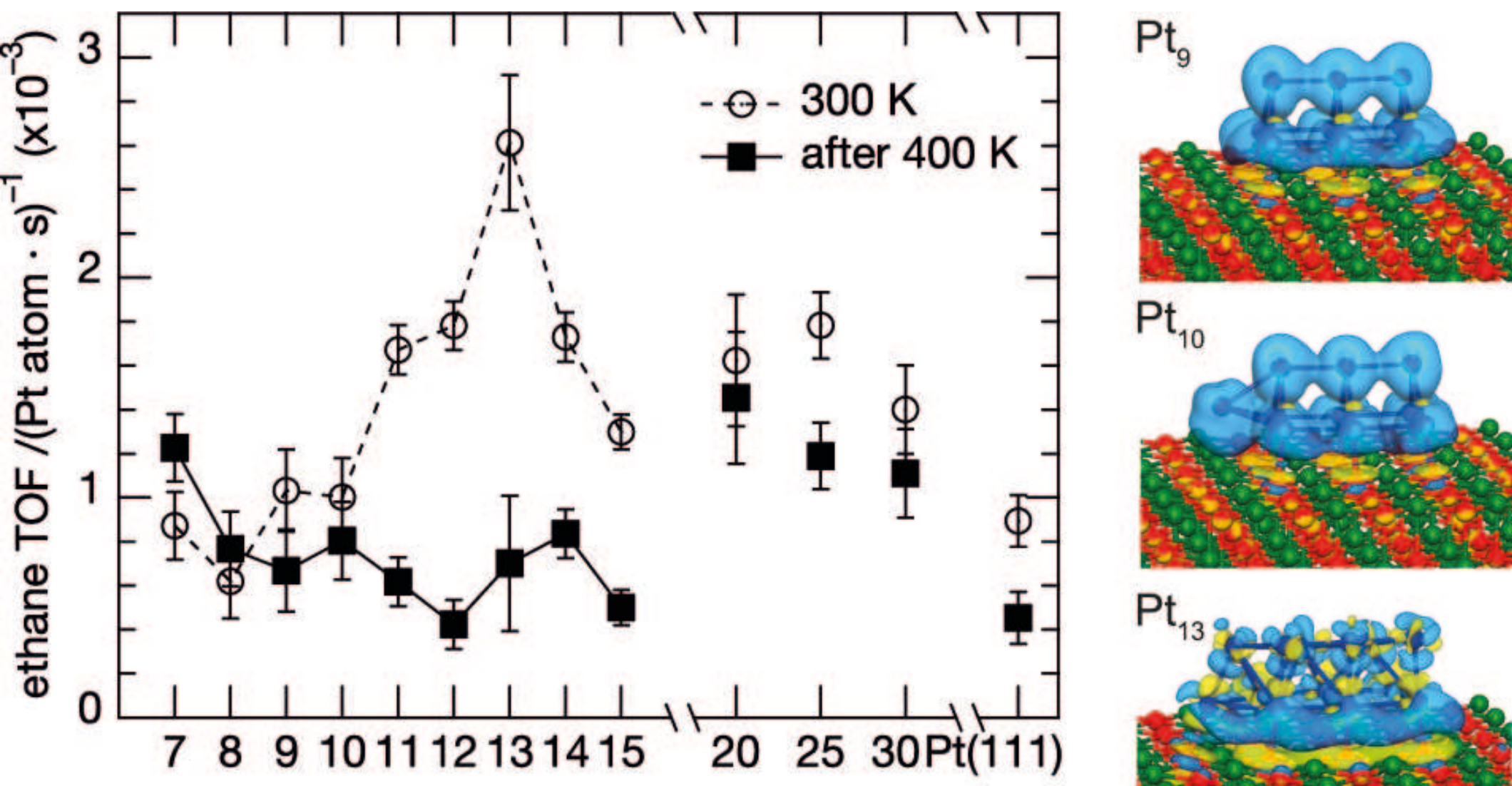

$\mathrm{Pt}_{10}$ number of atoms per cluster 